\title{
Nanotheranostics
}

2017; 1(1): 114-130. doi: 10.7150/ntno.18292

Review

\section{Heparin-Regulated Prodrug-Type Macromolecular Theranostic Systems for Cancer Therapy}

\author{
Huiyuan Wang1, Cheol Moon², Meong Cheol Shin², Yaping Wang1,4, Huining He4, Victor C. Yang4,5, \\ Yongzhuo Huang 1 四 \\ 1. Shanghai Institute of Materia Medica, Chinese Academy of Sciences, Shanghai 201203, China \\ 2. College of Pharmacy, Sunchon National University, Republic of Korea \\ 3. College of Pharmacy and Research Institute of Pharmaceutical Sciences, Gyeongsang National University, Gyeongnam, Republic of Korea \\ 4. Tianjin Key Laboratory on Technologies Enabling Development of Clinical Therapeutics and Diagnosis, School of Pharmacy, Tianjin Medical University \\ Tianjin 300070, China \\ 5. University of Michigan, College of Pharmacy, MI 48109-1065, USA
}

$\triangle$ Corresponding author: Yongzhuo Huang, Ph.D., Prof., Shanghai Institute of Materia Medica, Chinese Academy of Sciences, 501 Hai-ke Rd, Shanghai 201203, China. Tel: +86-21-2023-1000 ext 1401; Fax: +86-21-2023-1981; Email: yzhuang@simm.ac.cn.

(C) Ivyspring International Publisher. This is an open access article distributed under the terms of the Creative Commons Attribution (CC BY-NC) license (https://creativecommons.org/licenses/by-nc/4.0/). See http://ivyspring.com/terms for full terms and conditions.

Received: 2016.11.10; Accepted: 2017.01.13; Published: 2017.03.03

\begin{abstract}
Heparin is a kind of naturally occurring polymer with excellent biocompatibility and solubility. It is characterized by dense of negative charge, higher than any endogenous components. Heparin can bind with various cationic peptides and proteins, thereby providing a useful noncovalent linkage for building a drug delivery system. As a case in point, heparin/cell-penetrating peptides (CPP) interaction is strong, and remains stable in vivo. They can be used to modify different proteins, respectively, and subsequently, by simply mixing the modified proteins, a protein-protein conjugate can be form via the stable heparin/CPP linkage. This linkage could not be broken unless addition of protamine that bears higher cationic charge density than CPP, and CPP thus can be substituted and released. Of note, heparin is a potent antagonist of CPP, and their binding naturally inhibits CPP-mediated drug cell penetration. Based on this method, we developed a heparin-regulated macromolecular prodrug-type system, termed ATTEMPTS, for drug targeting delivery. In this review article, we mainly summary the application of ATTEMPTS in delivery of various macromolecular drugs for cancer therapy, and also introduce the heparin-regulated nanoprobes for tumor imaging.
\end{abstract}

Key words: Heparin, heparin-regulated macromolecular prodrug-type system, drug targeting delivery

\section{Functions and structure of heparin}

Polysaccharide-protein interactions play an important role in numerous physiological processes.[1] As a case in point, heparin-protein interactions have been widely investigated for better understanding their biological functions. Heparin, a linear polysaccharide, can bind to a variety of proteins (e.g. enzymes, growth factors, plasma proteins and extracellular matrix proteins), and regulate their activities and biological processes, such as cell signaling and morphogenesis.[2] Characteristics of various heparin-binding proteins were described in the review article.[3] These proteins generally possess the heparin-binding sites on the external surface and correspond to shallow packets of positive charge. The prominent type of heparin-protein interaction is ionic, but hydrogen bonding and hydrophobic interaction are involved as well in some cases. Such a heparin-based macromolecular interaction draws more and more attentions not only for its important role in understanding physiological and pathological processes, but also for its potential application in drug delivery.

Heparin is a highly sulfated glycosaminoglycan with a linear polymer structure consisting of repeated 
units of $1 \rightarrow 4$-linked pyranosyluronic acid and 2-amino-2deoxyglu-copyranose (glucosamine) residues (Fig. 1), which possesses the highest negative charge density of any known biological molecule.[3] Heparin is only produced in mast cells, and the commercial heparin is obtained from hog mucosa or bovine lung, with an average MW of 12-15 kDa.[4] The key features for heparin neutralization are anion-cation interactions as well as conformational flexibility of its linear structure.[5] Its unique biological and chemical properties render heparin broad medical application. Clinical use of heparin is an injectable anticoagulant via a mechanism that heparin binds to antithrombin III (AT) leading to its activation. The activated AT, in turn, inhibits thrombin and other involved proteases that attribute to blood clotting, yielding anticoagulant effect.

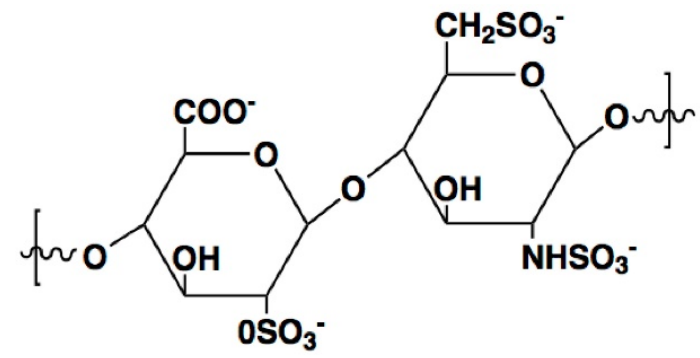

Fig. 1 The chemical structure of heparin unit.

Generally, heparin is capable of binding with peptides with basic amino acid residues. The largely electrostatic interaction of heparin and protamine is a well-studied example. Protamine, a highly cationic peptide rich in arginine, is a potent antagonist clinically used to heparin. Arginine appears to possess the strongest affinity to heparin among all amino acids owing to the ionic interaction, as well as the tight hydrogen bonding between the guanidinio group of arginine and a sulfo group in heparin.[3] Protamine firmly binds to heparin via a stable ion pair, with capability of neutralizing the free heparin and competitively binding to heparin and consequently disassociating the heparin/ AT complex. The heparin reversal can be achieved with an i.v. dose of $1 \mathrm{mg}$ protamine for every 100 USP heparin units.

\section{Non-covalent linkage via the interaction of heparin and cationic proteins / peptides}

Bioconjugation with antibody has been intensively investigated for creating a simple or complex structure of macromolecular drugs for targeted delivery, including chemotherapeutics, $[6,7]$ enzymes,[8] and nucleic acid drugs.[9] In an antibody-directed protein drug delivery strategy, covalent linkage is generally required for antibody-protein conjugation,[8] despite its pitfalls such as arduous chemical process and potential activity loss caused by crosslinking and steric block of protein active domains.

Reversible non-covalent linkage via the ionic interaction of heparin/protamine may provide a possible solution for overcoming the problems associated with chemical conjugation. The interaction between heparin-protamine is so strong and stable that it raised our initial interest for using as a non-covalent linkage for constructing bioconjugates. Because the binding of heparin/protamine is too stable to be reversed in vivo, however, a shorter cationic peptide with medium binding affinity to heparin is desired for this purpose. Based on this concept, we developed a strategy to derivate a protein drug with cationic species, i.e. an arginine-rich sequence, LMWP (low-molecular-weight protamine), rendering the protein bearing a heparin-binding domain, and thus the drug can non-covalently link to a heparin-modified antibody (Ab-heparin/peptidedeviated drug complex).

\section{Heparin-regulated Prodrug-type "ATTEMPTS" for drug delivery}

Electrostatic interactions have long since been used as a major mechanism for drug delivery. As a case in point, cationic carriers, binding with polyanionic nucleic acid drugs, has been widely investigated for gene therapy. Cationic carriers can facilitate cell-impermeable nucleic acid drugs, by forming reversible complexation through electrostatic binding mechanism, for intracellular delivery. The nucleic acid drugs will not take their therapeutic action until they are released from the polyelectrolyte complexes.[10] Thus, such complex can be viewed as a prodrug-type system, and the electrostatic interaction can be reversed by adding a competitively charged component.

Although prodrug approaches have been demonstrated to be clinically successful in chemical drugs,[11] such approaches are difficult finding their way in protein drug delivery. Due to the highly complicated 3-D structure of a protein, it would be far less feasible to block its active domain by using a promoiety with a reversible chemical linkage as simply done in chemical drugs. However, the abovementioned concept of heparin-assisted antibody directed protein drug delivery (Ab-heparin/peptidederivated drug) provides an approach to construct a macromolecular prodrug system via steric block the active domain. The attachment of a larger $\mathrm{Ab}$ molecule would create steric hindrance to the active domain of the protein drug, potentially blocking its 
activity. Such a steric hindrance, on the other hand, favorably intermits the drug action in the body, unless the linkage is broken and the drug released from the complex. Therefore, a strategic design of a breakable linkage is important.

Based on this approach, we developed an antibody targeted, triggered, electrically modified prodrug-type strategy, termed "ATTEMPTS", for macromolecular drug delivery. Herein several applications in protein drug delivery are presented.

\section{1. “ATTEMPTS” for targeting enzymatic therapy for thrombosis}

Thrombus formation is the underlying etiology in cardiovascular diseases, leading to the reduced blood flow to vital organs. One approach for treating thrombosis is to dissolve the blood clots with thrombolytic agents, namely plasminogen activators (PA). However, enzymatic therapy with PA often carries the risk of hemorrhage due to the excess activated plasmin in circulation. To address this problem, we used heparin-assisted "ATTEMPTS" for site-specific delivery of PA targeting to fibrin blots with reduced risk of hemorrhage.[12, 13] The system is comprised of a protein conjugate made of two parts (Fig. 2):

Part I. a fibrin-targeting antibody (anti-fi $\mathrm{Ab}$ ) linked with anionic heparin, and

Part II. a plasminogen activator (PA) derivatized with a cationic species.

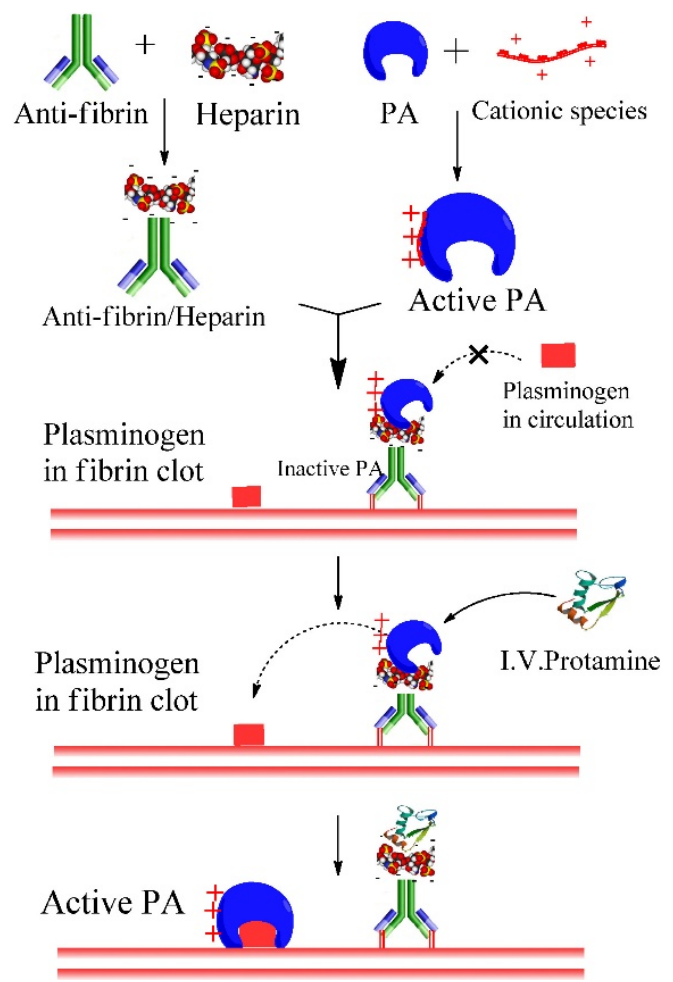

Fig. 2 Heparin-assisted "ATTEMPTS" for site-specific delivery of PA targeting to fibrin blots with reduced risk of hemorrhage.
These two parts can self-assemble through electrostatic interaction of heparin and the cationic peptide. Since the cationic peptide is relatively small, the derivatized PA can retain its thrombolytic activity, which however would be inhibited after binding with the Ab-heparin due to its steric blockage to the PA's active site. Such a self-assembled complex can be effectively dissociated by adding protamine, a clinical heparin antagonist that competitively binds with Ab-heparin, thereby allowing release of the derivatized PA and triggering its thrombolytic action. [14-16]

There are two characters for this ATTEMPTS macromolecular prodrug system:

(1) Ab mediated targeting component Systemically administered PA exposes the received patients to a life-threatening risk of hemorrhage, which however could be reduced by fibrin targeting therapy. The fibrin-targeting component consists of anti-fibrin $\mathrm{Ab}$ chemically conjugated to heparin. For constructing this targeting component, a synthesis method was meticulously selected in order to retain the original functions of both anti-fibrin $\mathrm{Ab}$ and heparin. The reducing end of heparin was site-specifically linked to the sugar moieties on the Fc region of the $\mathrm{Ab}$. Both heparin and $\mathrm{Ab}$ in the conjugates demonstrated $>85 \%$ of their original binding capacity to the cation-modified tPA and fibrin clot was retained.[15]

(2) Drug component A cationic species, typically a small cationic peptide less than $2 \mathrm{kDa}$, was used to prepared the derivatized drug. Such size is a result of careful consideration. First, short peptide would not sterically block the t-PA's active domain. Second, the cationic peptide should possess high affinity to heparin-Ab to form the complex that may sustain the complicated systemic delivery without dissociation. However, this affinity should be weaker than that between heparin and protamine so that the thrombolytic activity would be fully recovered when given the external trigger, protamine. It is a crucial part for the macromolecular prodrug system.

A polyarginine peptide, namely $(\mathrm{Arg})_{7}$, was chemically conjugated to tPA, while anti-fibrin antibody was modified with heparin. These two components then bind together in a non-covalent form - $t P A-(\text { Arg })_{7} /$ heparin-Ab. [14] A hypothesis of the ATTEMPTS is that the $\mathrm{Ab}$ component not only performs a targeting function, but also works as pro-moiety to sterically inhibit the activity of tPA, turning it into a prodrug state. The electrostatic interaction between $t P A-(A r g)_{7}$ and heparin- $A b$ would be reversed by adding protamine, thus reactivating tPA. The in vitro study showed that the activity of tPA was reduced to $40 \%$ in the complex of 
$t P A-(\text { Arg })_{7} /$ heparin- $A b$, and could fully recover after triggering with protamine (Fig. 3A).

In addition, a recombinant DNA method was explored to produce a cationic tPA by inserting a DNA fragment encoding a cationic peptide sequence to the tPA plasmid. $[15,17,18]$ Compared to chemical method, the recombinant fusion cationic tPA offers a homogeneous product, and importantly, with a benefit of achieving site-specific introduction of the cationic peptide. The recombinant cationic tPA displayed equal function in either the binding ability to heparin- $\mathrm{Ab}$ and the reversibility triggered by protamine. The in vitro fibrin clot assay showed that the heparin-bound tPA (well 6) exhibited a significantly less activity than free tPA, and the clot lysis activity, however, was recovered by addition of protamine (Fig. 3B).

In the conventional prodrug approaches, activation of the parent drugs generally undergoes chemical changes in structure, responding to the metabolic processes or the alternations in the microenvironments featured by the chemo-physical variety (e.g. low $\mathrm{pH}$ and overexpressed enzymes) from the normal tissue. The activation process would be slow and incomplete. Additionally, targeting to the upregulated signals (e.g. ROS and proteases) in cancer cells to trigger or activate offers another method to improve tumor delivery efficiency for therapy or imaging [19-23]. In contrast, the "ATTEMPTS" is not based on chemical reactions, but instead uses a competitive ionic mechanism of heparin-peptide interaction. Thus, a rapid onset of pharmacological activity can be achieved by the instant release of active drugs.

In vivo demonstration of thrombolytic efficacy was carried out on a rat thrombosis model, in which the inferior vena cava was ligated to yield a stasis-induced thrombus formation, with fibrin deposition in the aged clots. The complex of $t P A-(A r g)$ /heparin- $A b$ was given intravenously. After a predetermined period, protamine was i.v. injected to trigger the action of $\mathrm{tPA}$. Upon complexation of tPA with Hep-Ab, the thrombus dissolution was relatively insignificant, and comparable to the control, implicating that the fibrinolytic activity of tPA was inhibited (Fig. 4) [24]. Triggering release of tPA from the complex by protamine resulted in a lower clot weight than the control (Table 1), confirming the possibility of restoring the fibrinolytic activity by protamine administration.

As described above, the ATTEMPTS concept was developed for targeted and triggerable delivery for thrombolytic therapy. Based on the similar idea, another version was proposed, in which a "reversible camouflage" human serum albumin (HSA) around tPA was designed for a prodrug-type delivery (Fig. 5).[25] The tPA molecule is modified with low molecular weight heparin (LMWH), which electrostatically crosslinks with the protamine-conjugated HSA with targeting ligand. A major difference in this modified version is that the linkage is $\mathrm{LMWH} /$ protamine, compared to the previous of LMWP/heparin. Accordingly, the trigger agent for dissociation of $\mathrm{LMWH} /$ protamine complex is heparin, which competitively substitute LMWH out of the complex. A significant benefit of using heparin as trigger is that this FDA-approved drug is also part of the regimen for thrombolysis as well. The in vivo experiments in rat thrombosis model were carried out by injection of the drug systems. Heparin was administered at $15 \mathrm{~min}$ postdose to trigger the release of tPA at the target thrombus site. The results demonstrated that the camouflaged tPA system not only enhanced the clot lysis, but also reduced the bleeding risk (Fig. 6).
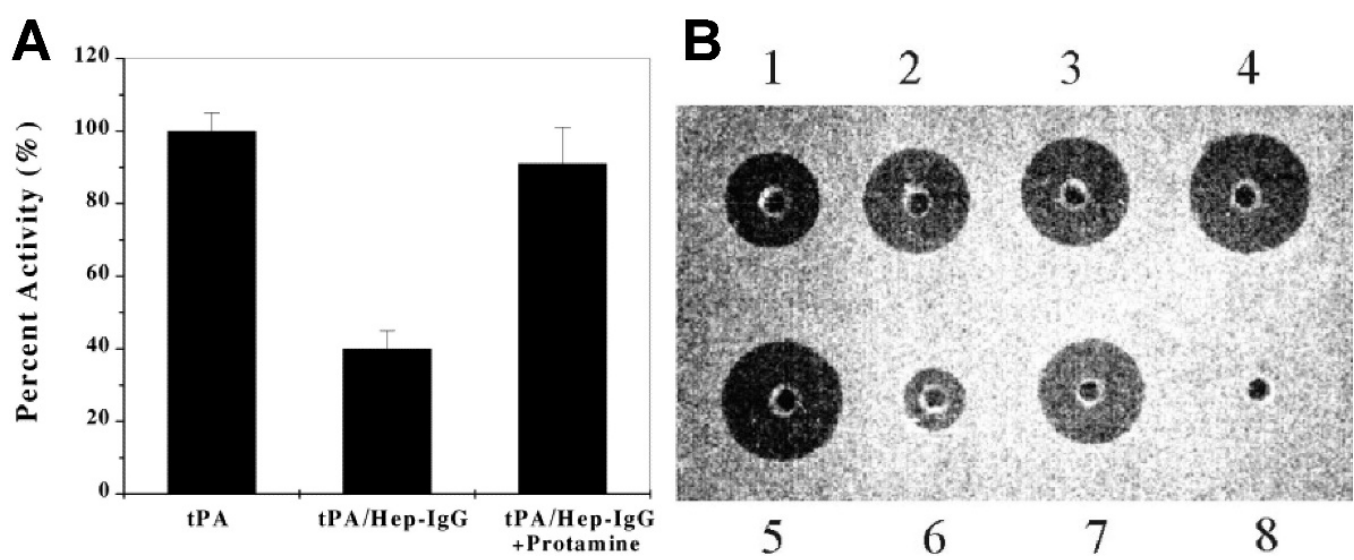

Fig. 3 (A) Inhibition of tPA activity by the heparin-antifibrin $\lg G$ and reversal by protamine. (B) In vitro clot lysis studies. Wells $1-4$ contain $0.025,0.05,0.1$ and $0.2 \mu g$ of tPA, respectively; well 5 contains $0.2 \mu \mathrm{g}$ of free recombinant cationic tPA; well 6 contains $0.2 \mu \mathrm{g}$ recombinant cationic tPA binding to the heparin complex (herein heparin beads were used); well 7 contains $0.2 \mu \mathrm{g}$ recombinant cationic tPA triggering with $50 \mu \mathrm{g}$ protamine; well 8 contains buffer only. Reproduced with permission from [14]. 


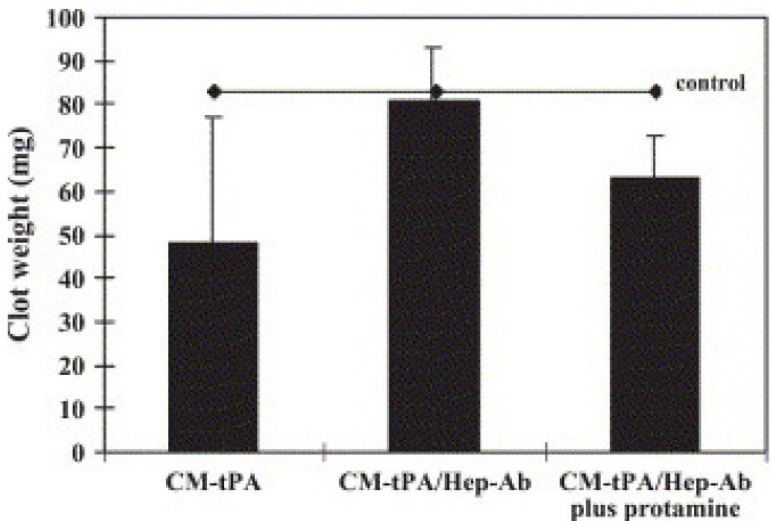

Fig. 4 Extent of clot dissolution after administration of CM-tPA, CM-tPA/Hep-Ab, or CM-tPA/Hep-Ab plus protamine. The inferior vena cava of each rat was harvested for determination of clot weight. Reproduced with permission from [24]. (Note: CM-tPA, cation-modified tPA)

Table 1. Clot weight after treatment of ATTEMPTS

\begin{tabular}{|c|c|}
\hline Treatment & Clot weight (mg) \\
\hline Saline (control) & $82.7 \pm 13.2$ \\
\hline$(\mathrm{Arg})_{7-\mathrm{tPA}}$ & $48.3 \pm 11.5$ \\
\hline$t P A-(\text { Arg })_{7} /$ heparin $-A b$ & $80.6 \pm 12.2$ \\
\hline tPA-(Arg) $)_{7}$ heparin-Ab + protamine & $62.9 \pm 9.9$ \\
\hline tPA (commercial) & $55.9 \pm 14.5$ \\
\hline
\end{tabular}

\subsection{Heparin-assisted prodrug strategy for enhanced intracellular delivery with cell-penetrating peptide}

Protein drugs are rigidly limited from cell entry due to their large size and high hydrophilicity, even though they are successfully delivered to the target organs. Cell-penetrating peptides (CPP), short cationic peptides less than 20 amino acids, are one of the most powerful tools for protein cellular transduction via chemical conjugation or recombinant method.[26] CPP-medicated drug delivery has been intensively studied in the recent two decades, despite the lack of clear depiction of the cellular transduction mechanisms.[27] To our great interest, CPP is ideal suitable working as the cationic species in the "ATTEMPTS" due to its polycationic nature. The high affinity between CPP and heparin secures the formation of the complex (Ab-heparin/CPP-drug). The targeting component, via such electrostatic binding with heparin, is introduced into the delivery system for an active targeting function. More importantly, CPP has been confirmed its potent ability to deliver its attached cargos into all types of cells indiscriminately.

Moreover, CPP is also useful in tissue-penetrating drug delivery, efficiently overcoming the formidable barriers such as the blood-brain barrier (BBB),[28] nose-to-brain,[29] skin,[30, 31] and tumors with high interstitial pressure.[32] a

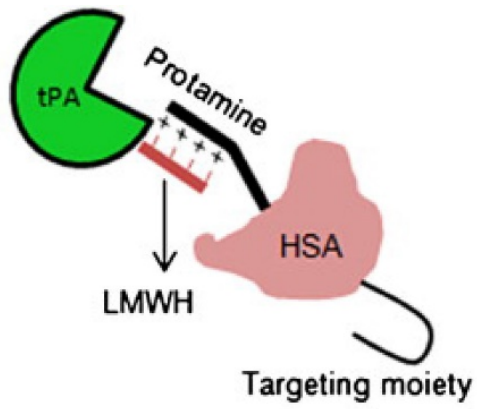

b
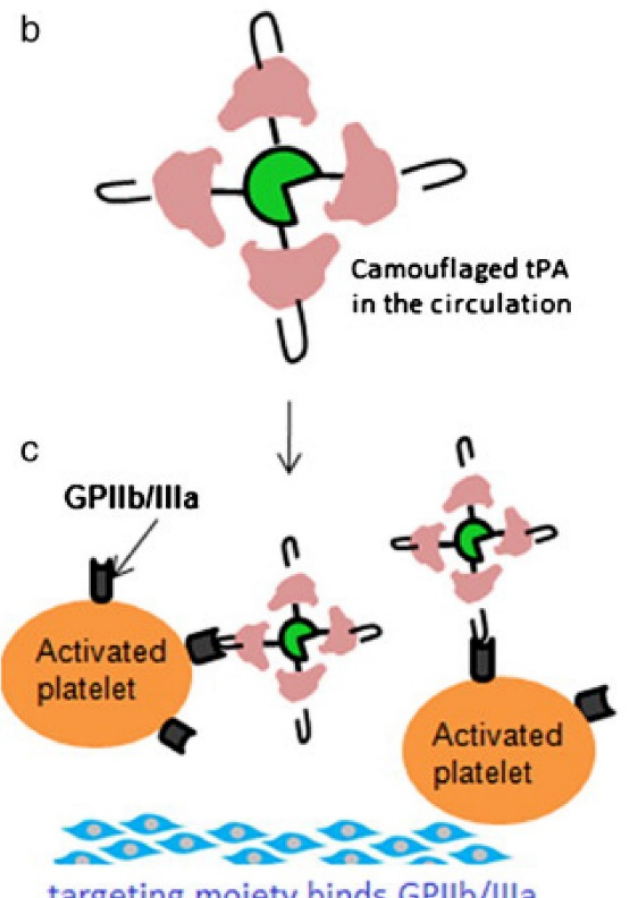

targeting moiety binds GPIIb/IIla
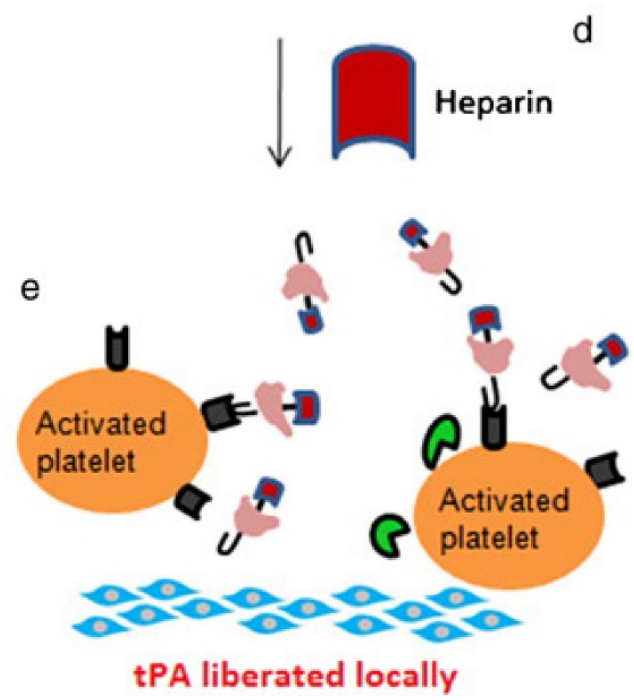

Fig. 5 (a) The construct of camouflaged tPA consisting of targeting peptide-HSA-protamine and tPA-low molecular-weight heparin (tPA-LMWH). (b) The albumin bound with tPA will provide steric hindrance to tPA-binding macromolecules in plasma. (c) Deposition of the complex on the surface of the activated platelets associated with the thrombus via peptide-glycoprotein (GP) Ilb/llla binding; (d) Administration of heparin after accumulation of the complex at thrombus site; (e) Triggered release of tPA for local plasminogen activation and fibrinolysis. Reproduced with permission from [25]. 


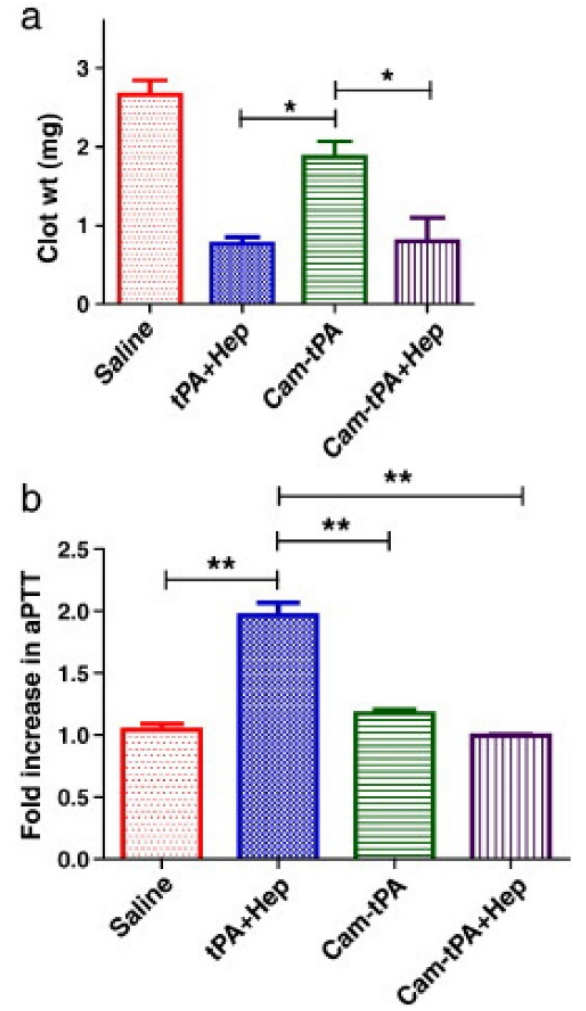

Fig. 6 In vivo clot lysis in rat jugular-vein thrombosis model. (a) Extent of clot lysis after administration of tPA and heparin combination (tPA-Hep), camouflaged tPA and camouflaged tPA plus heparin. (b) Fold increase in aPTT 1 $\mathrm{h}$ after the treatment compared to the value measured before the intervention. Data represent mean $\pm \mathrm{SD}, \mathrm{n}=3,{ }^{*} p<0.05,{ }^{*} p p<0.01$. Reproduced with permission from [25]

\subsubsection{CPP-heparan sulphate interaction}

Yet, the cell-penetration ability can be completely inhibited in the presence of heparin, heparan or dextran sulfate,[33] which blocks the interaction between CPP and the cell surface ligands. Electrostatic interaction is a key factor governing the first contact of cell-surface binding for CPP.[34] Typically, the cell surface is negatively charged, and cationic CPP binds non-specifically to the cell surface, thus initializing the cellular transduction process by adsorptive endocytosis.[35] The major binding site on cell surface for CPP is the negative heparan sulphate (HS) proteoglycans that are ubiquitously present on cell surfaces, with $10^{5}-10^{6}$ molecules per cell,[36] and the binding with $\mathrm{HS}$ is the first step for CPP-medicated cell entry. Removal of HS by heparinase would inhibit CPP internalization.[37] Heparan sulfate is a member of the glycosaminoglycan family of carbohydrates, an analog of heparin - both consist of sulfated repeating disaccharide unit. Due to their high similarity in structure, CPP also displays high affinity to heparin, and this property was exploited for purification of CPP-protein with a heparin affinity column.[38] However, a more interesting application of heparin is to work as an ideal antagonist of CPP because the addition of heparin would competitively inhibit the interaction between CPP and HS on cell surface and the consequent cell entry.[39, 40] Despite its potent cellular transduction of macromolecules, CPP is also known for its non-selective cell penetration, which often causes unwanted side effects on normal tissues.[41] However, it is a formidable challenge to steer the CPP-based system to its destination and manipulate its cell penetration ability, i.e. cell entry occurs only at the desired sites. The use of heparin as antagonist provides a promising means to control the nonselective CPP-medicated cell entry by reversibly blocking the interaction between CPP and HS.

\subsubsection{Heparin-regulated cell penetration}

Based on this feature, we developed an updated version of "ATTEMPTS" with a feature of triggerable "on-and-off" function of cell penetration for anticancer drug delivery (Fig. 7). The basic concept is similar, and the system consists of two parts: targeting component (Ab-heparin) and drug component (CPP-drug). The important function of CPP is that it plays dual roles in this modified system: (1) as the cationic species for electrostatic linker binding with the Ab-heparin, and (2) as a cellular transducer for its attached macromolecular cargo. The heparin-binding process curbs the nonspecific cell penetration ability of CPP by electrostatically neutralizing the positive charge of CPP.

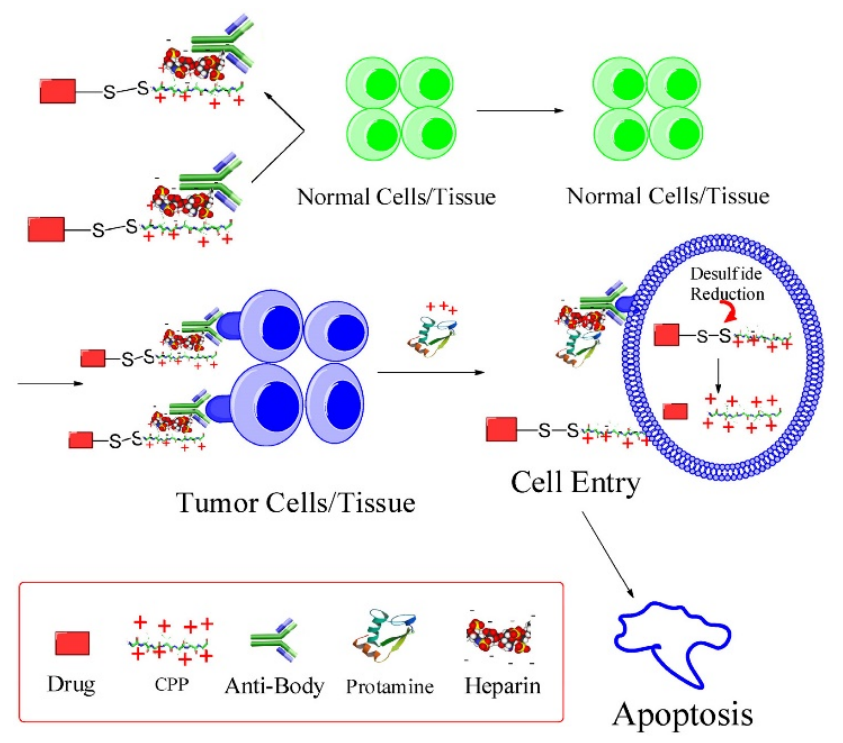

Fig. 7 The modified ATTMEPTS with a triggerable cell-penetrating ability. The system consists of a targeting component (Ab-heparin) and drug component (drug-CPP), which spontaneously associate with each other via the interaction of heparin-CPP. Following administration, the prodrug feature and targeting function can reduce non-specific cell penetration and alleviate side effects on normal tissues. In the targeted tumor, drug component would be released by triggering with protamine, and its cell-penetrating ability recovered. The cleavable S-S linkage was designed in favor of retaining the protein drug inside the cytosol. Reproduced with permission from [26]. 
For instance, low molecular weight protamine (LMWP) was firstly identified by our group via enzymatic digestion method,[42] and initially used as a substitute of protamine. LMWP, with sequence of VSRRRRRRGGRRRR, was later found that it bears potent cell penetration ability, and are able to mediate delivery of peptides,[43] proteins,[31, 44] gene,[44, 45] and nanoparticles.[28, 46] More importantly, it safety has been undergone scrutiny investigation both in vitro and in vivo (using dogs).[47, 48] Therefore, LMWP has been explored as both a CPP and a heparin-binding ligand in the prodrug-type delivery system design.

It should be pointed out that, however, heparin also possesses a strong affinity to plasma antithrombin III (AT $\mathrm{AII}_{\mathrm{II}}$, a mechanism that accounts for its anti-clotting function. Therefore, a selected CPP must possess stronger affinity to heparin than $\mathrm{AT}_{\text {III }}$ so that the $\mathrm{CPP} /$ heparin linkage could remain stable in vivo. Our previous results showed that $\mathrm{AT}_{\text {III }}$ detached from the heparin affinity column at a high-salt elution of $0.6 \mathrm{M} \mathrm{NaCl}$, while LMWP at $0.94 \mathrm{M} \mathrm{NaCl}$ and protamine at $1.3 \mathrm{M} \mathrm{NaCl}$.[18] Hence, LMWP fits neatly into the elution frame, with a much stronger affinity to heparin than $\mathrm{AT}_{\text {III }}$, but less than protamine. Protamine thus works ideally as a trigger for releasing CPP-drug from the complex and fully recovering its cell penetration ability. Such strategic selection secures Ab-heparin/CPP-drug maintaining a stable complex in blood stream, unless protamine is given to trigger the dissociation.

Cell penetration is a key factor determining the therapeutic effect of protein toxins that target subcellular organelles. In this sense, cell impermeability of the native proteins can be considered as an inactive form, whereas the CPP-modified counterparts are pharmacologically active because of their ability to cell penetration. Thus, the cell penetration functionality is associated with the pharmacological activity that can be "switched" on/off. Based on this principle, a macromolecular prodrug could be developed. For example, gelonin, a plant protein toxin with potent anticancer activity in cytosol, yields unproductive treatment outcomes due to its poor cellular uptake. However, the naturally membrane-impermeable protein toxin can be turned into a cell-penetrating drug by modification with LMWP via either chemical or recombinant method. Accordingly, the cytotoxicity of LMWP-modified gelonin against tumor cells was significantly enhanced owing to its acquired cell-penetrating ability. The IC50 of LMWP-gelonin was 20-120 folds lower than that of gelonin on various tumor cells (Table 2).[49] Similar cell penetration ability was observed in TAT-modified gelonin (TAT-Gel), which also yielded significantly augmented cytotoxicity in various cells (229-fold for LS174T; 391-fold for HCT116; 93-fold for MDCK and 108-fold for 293 HEK) than gelonin.[50]

However, the cell penetration ability of the CPP-gelonin was suppressed by addition of the CPP antagonist, heparin. The CPP-gelonin was inhibited from cell entry and remained inactive extracellularly, unless triggering on a recovery by protamine, which competitively binds with heparin and thus releases the CPP-gelonin (Fig. 8).[51]

Table 2. IC50 values of $r G e l, c G-L$ and $r G-L$ in various cancer cell lines. [49]

\begin{tabular}{lllll}
\hline & CT26 $^{\mathrm{a}}$ & LS174 $^{\mathrm{a}}$ & 9L $^{\mathrm{a}}$ & PC-3a \\
\hline rGel & $1630 \pm 510$ & $5870 \pm 1030$ & $3100 \pm 420$ & $3430 \pm 1140$ \\
cG-L & $37.3 \pm 15.7^{* * *}$ & $123.1 \pm 26.9^{* * *}$ & $106.2 \pm 22.7^{* * *}$ & $57.8 \pm 23.4^{* *}$ \\
rG-L & $79.1 \pm 21.4^{* * *}$ & $67.8 \pm 13.7^{* * *}$ & $61.3 \pm 12.2^{* * *}$ & $83.6 \pm 28.3^{* *}$ \\
\hline
\end{tabular}

For all experiments, $\mathrm{N}=3$. (rGel: recombinant gelonin, cG-L: gelonin-LMWP chemical conjugate, $\mathrm{rg}$-l recombinant gelonin-LMWP chimera).

a IC50 values are displayed as nM.

** $\mathrm{P}<0.001$.

$* * * \mathrm{P}<0.0001$

\section{A $\mathrm{rGel}$}
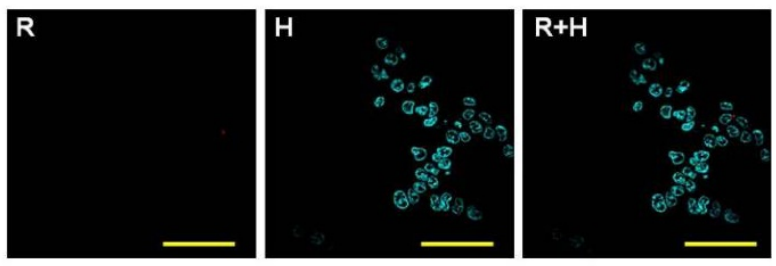

\section{B TAT-Gel}
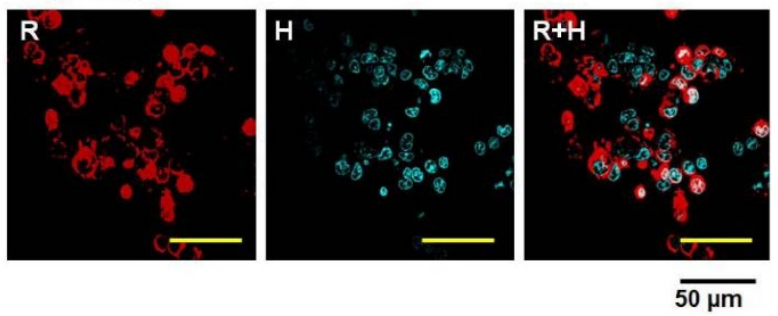

Fig. 8 Cell uptake study results of rGel and TAT-Gel on LS174T cells. The cells were incubated with either TRITC-labeled (A) rGel or (B) TAT-Gel for $3 \mathrm{~h}$ at $37^{\circ} \mathrm{C}$. After incubation, the cells were washed for three times with $10 \mathrm{mg} / \mathrm{mL}$ heparin/PBS solution for stringent wash, counterstained the nuclei with Hoechst 33342, and, after three more wash with PBS, the cell images were taken by a confocal microscope utilizing TRITC (red), Hoechst (blue) channels and merged. (rGel: recombinant gelonin, TAT-Gel: recombinant TAT-gelonin fusion protein). Reproduced with permission from [51].

Carcinoembryonic antigen (CEA) is a well-documented colon cancer cell biomarker, and has been widely used for targeted delivery.[52, 53] T84.66, a monoclonal anti-CEA antibody, is sensitive and selective to determine the presence of tumor in mice.[54] Therefore, T84.66 was used to construct a heparin-linked antibody (T84.66-heparin). The 
ATTEMPTS system of T84.66-heparin/TAT-gelonin was formed via the stable interaction between heparin/TAT, and its function was investigated in the CEA-overexpressing colon cancer cell line LS174T. In the presence of heparin, TAT-gelonin displayed diminished cytotoxicity (Fig.9A). [55] Similarly, the T84.66-heparin/TAT-gelonin was also pharmacologically inactive (Fig. 9B). Yet it could be activated by addition of protamine, and the released TAT-gelonin efficiently killed the tumor cells. When the molar ratio of heparin/protamine was larger than 5:1, the activity was completely restored, with an IC50 value similar to that of TAT-Gel, demonstrating the success of the heparin/protamine regulating ATTEMPT strategy (Fig. 9C).

In vivo treatment revealed that the highest antitumor efficacy could be achieved using the ATTEMPTS system along with i.v. injection of the trigger protamine (Fig. 10A-B). The results suggested the treatment could benefit from anti-CEA-directed tumor delivery and protamine-triggered release of TAT-gelonin. The group receiving T84.66-Hep/TAT-gelonin but without protamine displayed enhanced treatment compared to TAT-gelonin, probably due to the T84.66-mediated tumor targeting and accumulation, as well as T84.66-mediated endocytosis.

It should be pointed out that site toxicity (e.g., body weight loss) was observed in the group receiving T84.66-Hep/TAT-gelonin and protamine administration (Fig. 10C), probably caused by the non-specific activation of prodrug in blood stream and healthy organs. Therefore, the timing of protamine administration should be be further optimized.

\subsection{Heparin-regulated CPP-modified delivery system for enhanced asparaginase therapy}

L-asparaginase (ASNase) is an FDA approved enzyme drug for treating acute lymphoblastic leukemia (ALL) via a mechanism of hydrolysis of asparagine[56]. The consequent depletion of circulating asparagine leads to apoptosis of leukemic cells that must rely on the extracellular supply of asparagine for protein biosynthesis. We proposed a modified "ATTEMPTS" that intended to specially deliver ASNase into leukemic cells to eliminate the influx asparagine, with a benefit of avoiding systemic depletion of the essential nutrient asparagine from circulation $[57,58]$.

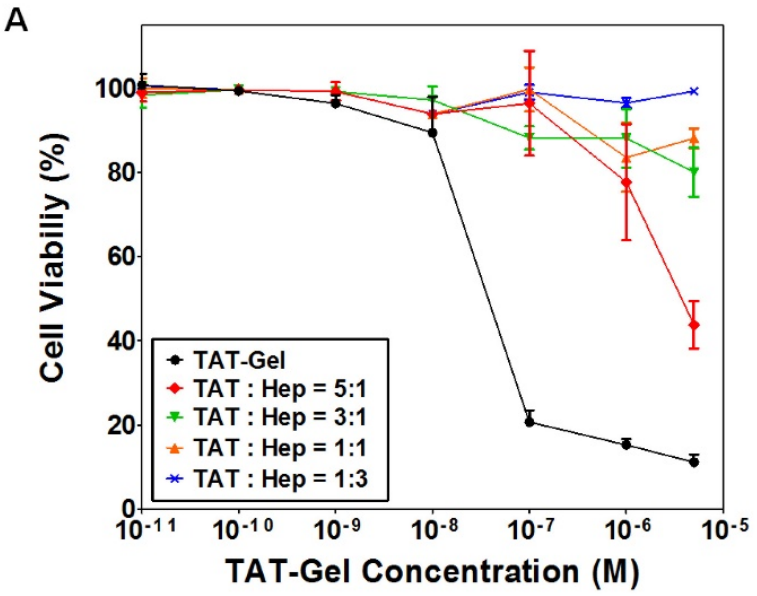

B

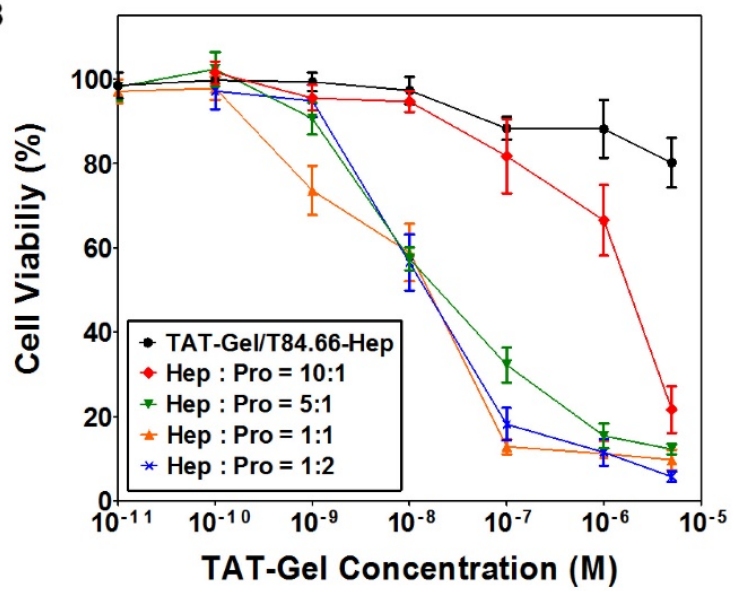

C

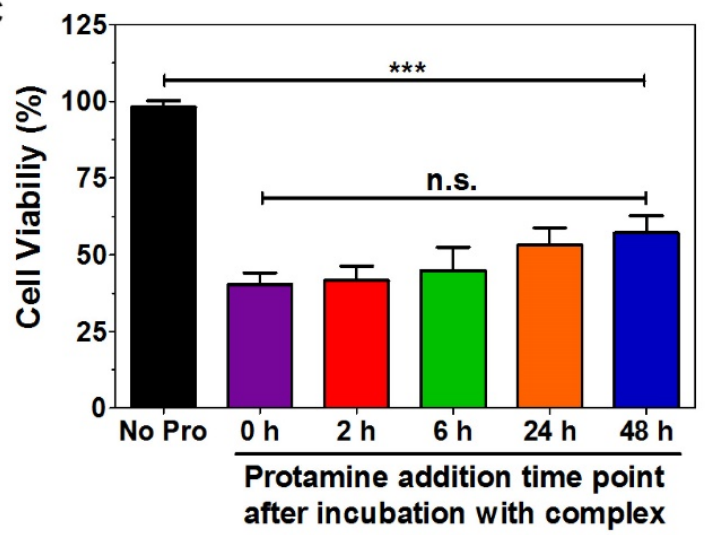

Fig. 9 T84.66-Hep/protamine-mediated modulation of cytotoxicity by TAT-Gel. (A) T84.66-Hep blocking of TAT-Gel cell transduction. When LS174T cells were incubated with TAT-Gel/T84.66-Hep complex, prepared by mixing TAT-Gel with increasing TAT-to-Hep molar ratios (from 5:1 to 1:3) of T84.66-Hep, a significantly reduced cytotoxicity was observed, compared with that of TAT-Gel. (B) Protamine-induced release of TAT-Gel. Addition of protamine to TAT-Gel/T84.66-Hep-treated cells, with increasing Hep-to-Pro molar ratios (from 10:1 to 1:2), cytotoxicity effects were significantly augmented. At above 5:1 molar ratio, the anti-cancer effect (IC50) was similar to that induced by TAT-Gel alone. (C) Time dependency of the protamine addition time on effects of protamine-triggered release. Cells were incubated with TAT-Gel/T84.66-Hep for $6 \mathrm{~h}$, and, after wash, at intended time points (0, 2 , 6, 24 and $48 \mathrm{~h}$ ), protamine (Hep : Pro molar ratio of 1:2) was added to the wells, and the cells were incubated with protamine further up to total $72 \mathrm{~h}$. ***P < 0.001 and n.s.: not significant by 1-way ANOVA (Tukey's multiple comparison test as the post hoc test). (TAT-Gel: recombinant TAT-gelonin fusion chimera, T84.66-Hep: T84.66-heparin chemical conjugate, Hep: heparin, Pro: protamine). Reproduced with permission from [55]. 
A

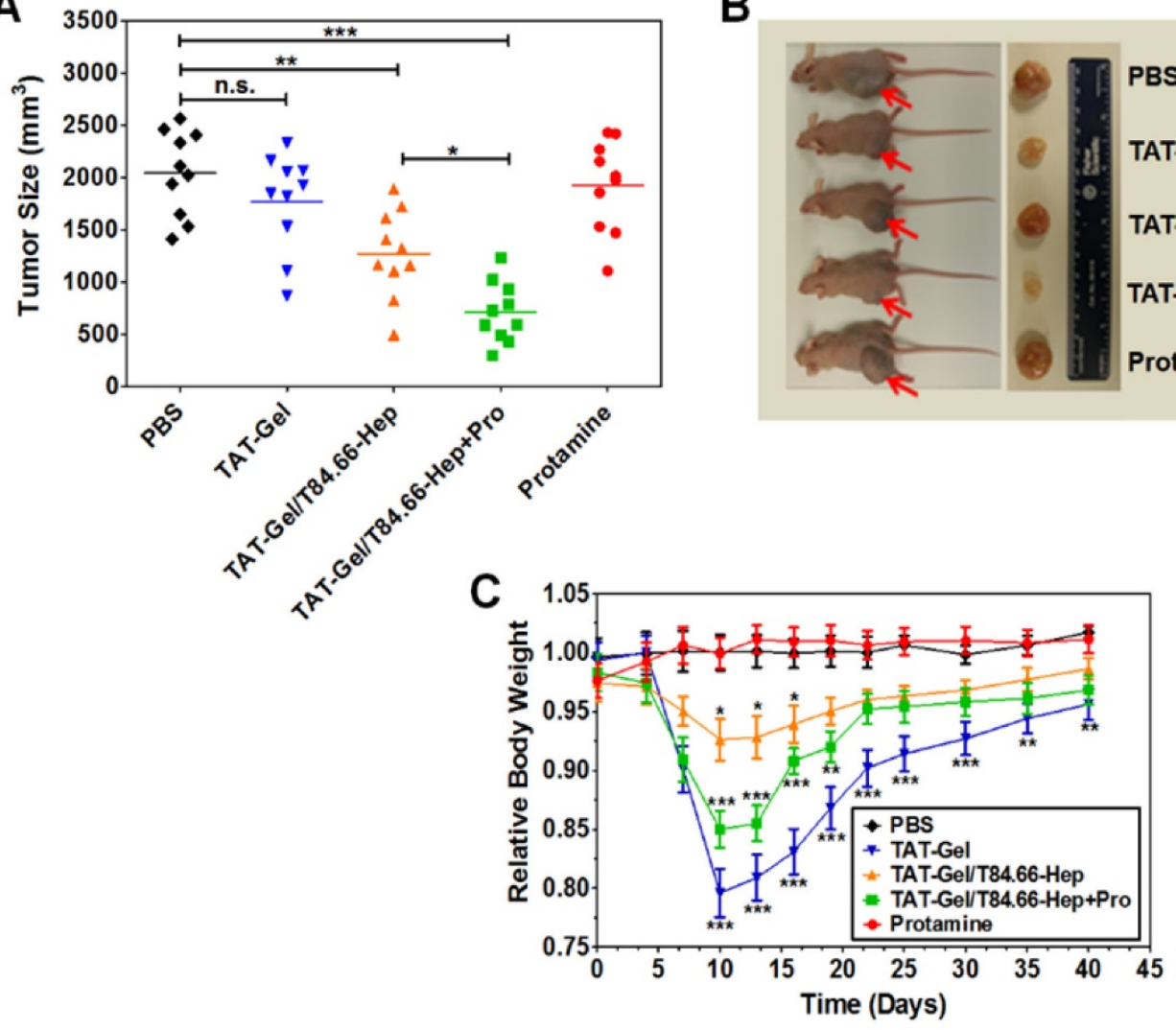

Fig. 10 In vivo proof-of-concept efficacy study for assessment of the feasibility of PTD-modified ATTEMPTS using LS174T xenograft tumor bearing mice. (A) Tumor volumes $(\mathrm{mm} 3)$ at day 40 (40 days after tumor implantation). At day 3, mice were divided into 5 groups $(\mathrm{N}=10)$ and treated with either: 1) PBS, 2) TAT-Gel, 3) TAT-Gel/T84.66-Hep, 4) “TAT-Gel/T84.66-Hep+Pro" or 5) protamine (a.k.a. Pro) for three times (at day 3, 6 and 9) via tail vein injection. (B) Representative mice and tumor images at day 40. (C) Relative average body weight change (\%) of mice during efficacy study. "TAT-Gel/T84.66-Hep+Pro" treatment exerted significantly enhanced therapeutic effects (*P<0.05), compared with TAT-Gel/T84.66-Hep treatment, yet induced higher toxicity. *P $<0.05$, **P $<0.01$ and ***P $<0.001$ by 1 -way ANOVA (Tukey's multiple comparison test as the post hoc test). (TAT-Gel: recombinant TAT-gelonin fusion chimera, T84.66-Hep: T84.66-heparin chemical conjugate, TAT-Gel/T84.66-Hep+Pro": treatment with TAT-Gel/T84.66-Hep complex with protamine). Reproduced with permission from [55].

The originally membrane-impermeable ASNase was conjugated to TAT peptide via a disulfide bond. An advantage of using S-S linkage is that after cleavage of the disulfide bond by the elevated level of glutathione in the cytosol, the protein turns back to be membrane-impermeable and thus is entrapped inside the cell. Cell penetration ability of the ASNase-TAT was investigated on the HeLa cells. According to the confocal studies, ASNase-TAT treated cells displayed significant higher cellular uptake efficiency than those treated with ASNase (Fig. 11). Indeed, the latter displayed virtually no fluorescence, indicating the incapability of ASNase in cell translocation. The heparin-regulated prodrug feature was demonstrated, in which ASNase-TAT was rejected from cell entry in the presence of heparin due to the complexation of ASNase-TAT/heparin, and its cell penetration ability was fully reactivated by addition of protamine (Fig. 11C \& D). These results were further confirmed by a separate experiment using human leukemia MOLT-4 cells and a quantitative flow cytometry assay (Fig. 12).
A

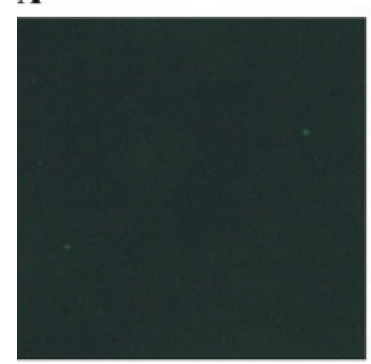

C

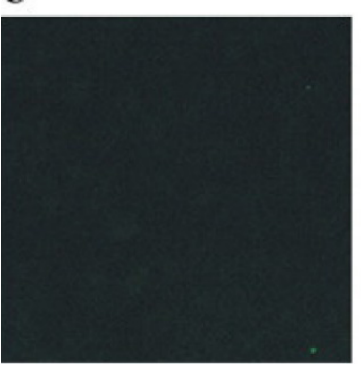

B

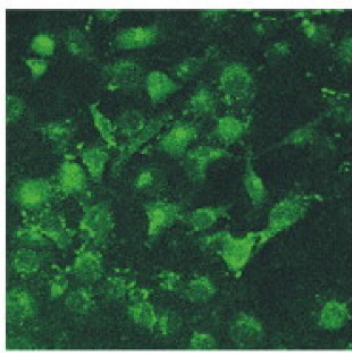

D

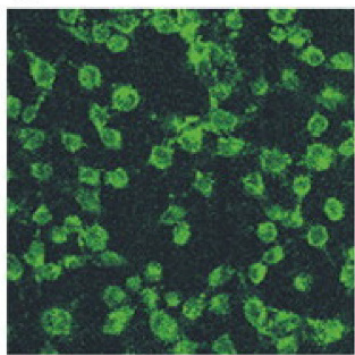

Fig. 11 Fluorescence microscopy of: (A) FITC-ASNase; (B) FITC-TAT-ASNase; (C) FITC-TAT-ASNase with heparin; and (D) FITC-TAT-ASNase with heparin and protamine. HeLa cells were incubated with the different treatment groups. Reproduced with permission from [57]. 


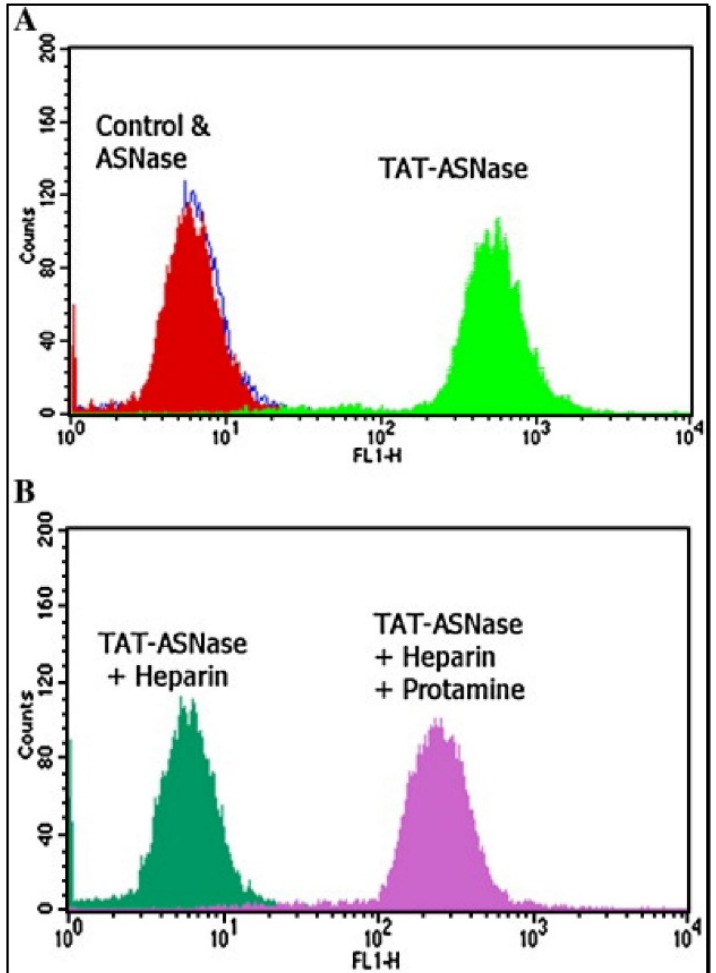

Fig. 12 Flow cytometry analysis of FITC-TAT-ASNase conjugate. (A) ASNase and TAT-ASNase were FITC-labeled and incubated with MOLT-4 cells. (B) FITC-TAT-ASNase was incubated with heparin or heparin and protamine in MOLT-4 cells. Reproduced with permission from [57].

\subsection{Heparin regulated CPP-modified delivery system for enhanced doxorubicin delivery}

A major problem for small anticancer drugs is the non-selective accumulation in both tumors and normal tissues, posing a severe limitation for clinical application. By contrast, macromolecules display unique advantages on favorably diffusing into tumors via passive accumulation owing to the leaky vasculature and impaired lymphatic drainage in solid tumors, a phenomenon termed the enhanced permeability and retention (EPR) effect.[59] Polymer-drug conjugation is widely used to convert a small drug into a macromolecule in order to improve PK profiles.[60] Despite such benefit, the attached hydrophilic polymer (e.g. PEG) is an unfavorable factor against cellular uptake.

To address this dilemma, "ATTEMPTS" was adapted, whereby the polymer-drug conjugate was modified with CPP to enhance it intracellular delivery, yet with a prodrug feature that CPP would be shielded by heparin unless triggering with protamine.[61, 62] A model anticancer drug (camptothecin or doxorubicin) was used for the studies. The CPP-modified polymer-drug conjugate (e.g. LMWP-polyrotaxane-camptothecin, LMWP-PR-CPT) was synthesized (Fig. 13).

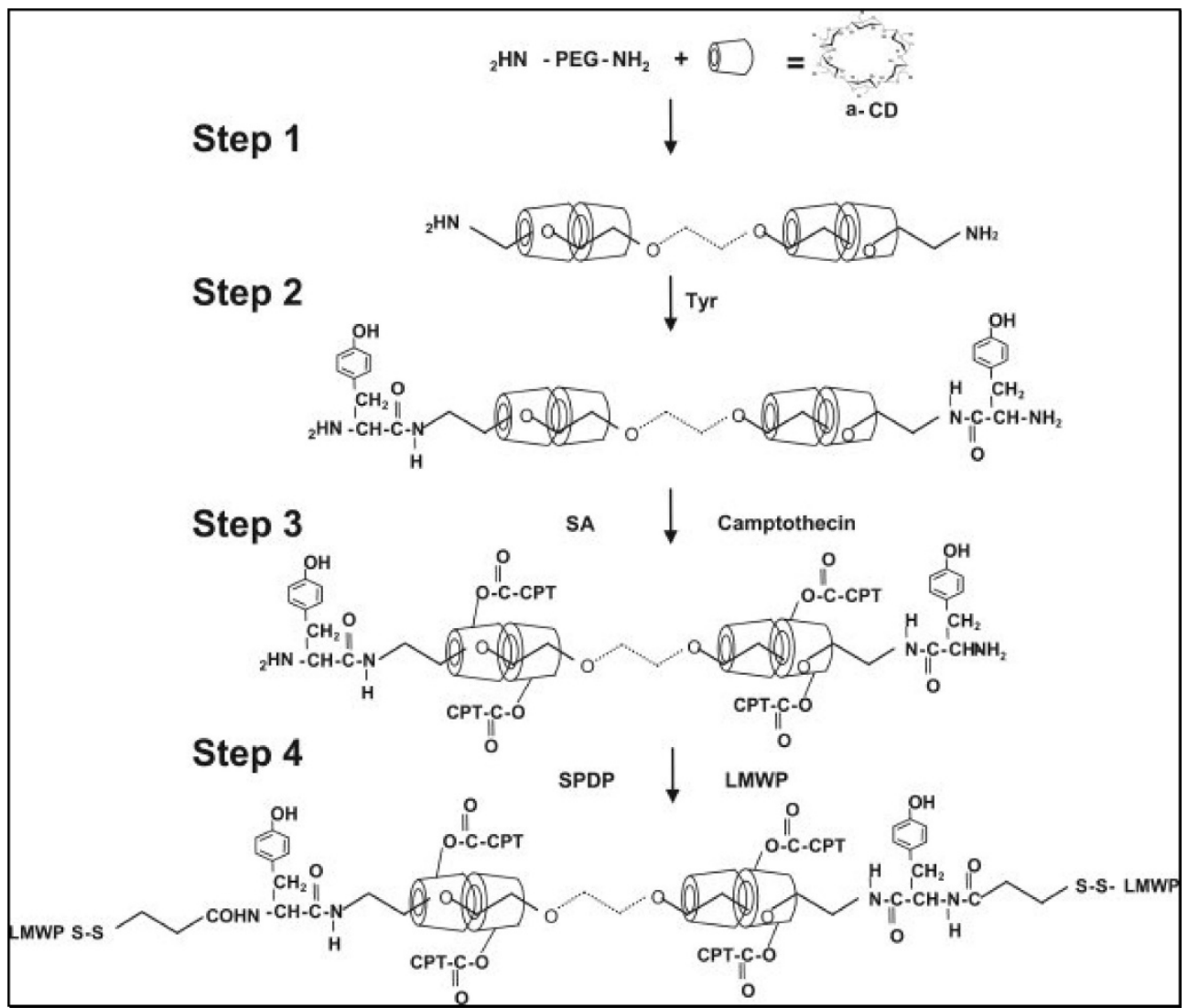

Fig. 13 Schematic illustration of the synthesis of CPT-PR-LMWP conjugates. Reproduced with permission from [61]. 
The PR-CPT conjugate was unable to be internalized into cells due to the macromolecular structure of the hydrophilic polymer, but was turned to be cell-permeable with modification of LMWP. Upon complexation with heparin, the cell penetration ability of LMWP-PR-CPT was abolished, but restored by disassociation from heparin with protamine. The macromolecular system would preferably accumulate in tumor via a passive targeting mechanism, i.e. EPR effect, thereby alleviating the non-specific toxicity. The intra-tumor heparin/LMWP-PR-CPT complex can be activated with protamine, and the intracellular delivery of LMWP-PR-CPT then triggered. CPT molecules would be released from the PR polymer by hydrolysis, killing the tumor cells. In tumor cellular study, the prodrug feature based on heparin regulation was demonstrated (Fig. 14).
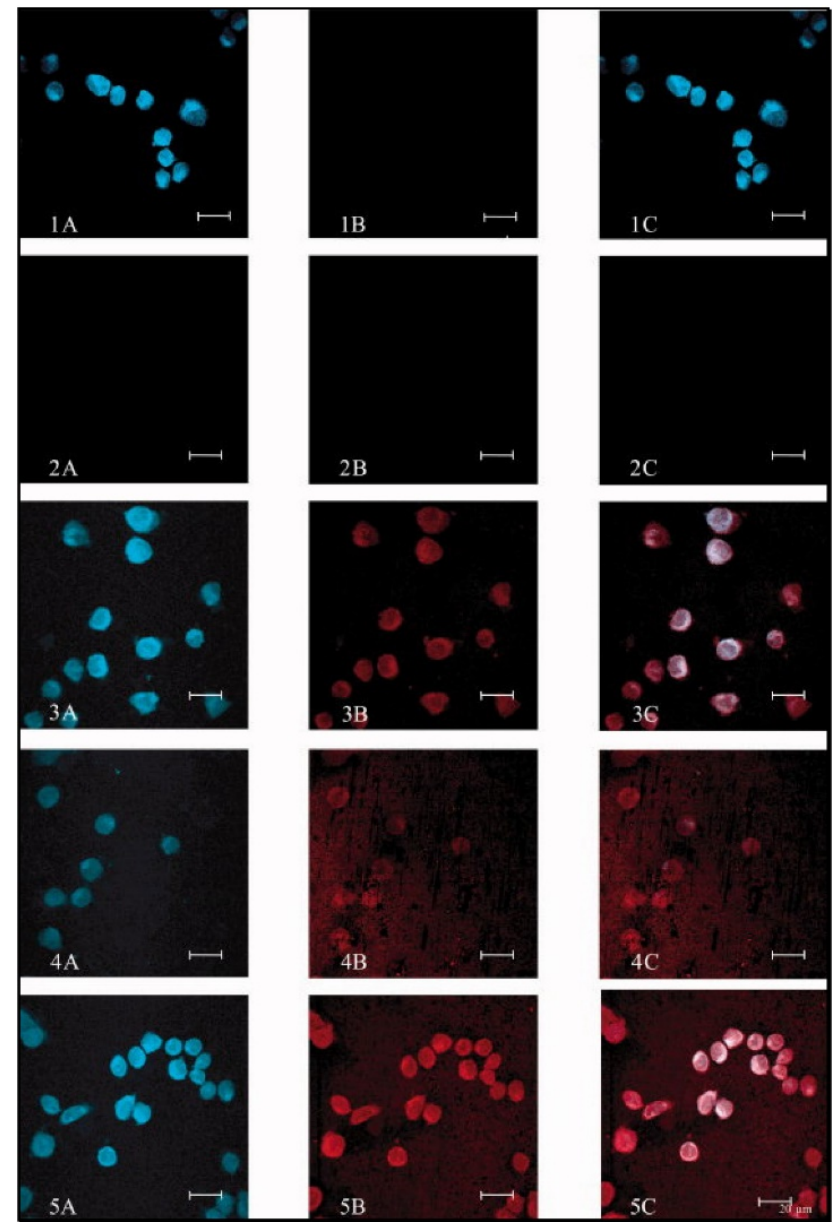

Fig. 14 Cellular localization of Rho-labeled LMWP-drug conjugates in A2780 human ovarian carcinoma cells. Free CPT, Rho-labeled CPT-PR, Rho-labeled CPT-PR-LMWP, Rho-labeled CPT-PR-LMWP and heparin mixture, and Rho-labeled CPT-PR-LMWP, heparin, and protamine mixture were overlaid onto cultured A2780 cells in the presence of $10 \%$ FBS. Cellular localization was monitored by confocal microscopy. (1) Free CPT; (2) Rho-labeled CPT-PR; (3) Rho-labeled CPT-PR-LMWP; (4) Rho-labeled CPT-PR-LMWP and heparin mixture; (5) Rho-labeled CPT-PR-LMWP, heparin, and protamine mixture. (A) $430 \mathrm{~nm}$ (blue) detection; (B) $560 \mathrm{~nm}$ (red) detection; $(C)$ overlaid $(A+B)$. Reproduced with permission from [61].

\section{Heparin-drug conjugates as prodrugs}

Heparin is a highly water soluble polymer, and it is often used as a drug carrier to increase drug solubility. Moreover, with application of cleavable linkers, the heparin-drug conjugates can be turned into macromolecular prodrugs. For instance, the amphiphilic heparin-paclitaxel prodrug was synthesized by reacting paclitaxel with succinylated-heparin via a single amino acid spacer.[63] This prodrug-like system could self-assemble and form nanoparticles with a hydrophobic paclitaxel core and heparin shell. The prodrug with leucine spacer was subjected to hydrolysis of the ester bond between the amino acid and paclitaxel, thus releasing the parent paclitaxel drug. The cytotoxicity results showed the IC50 of the prodrug was three-fold lower than that of free paclitaxel $(0.058$ vs $0.19 \mu \mathrm{g} / \mathrm{mL})$. Although the prodrug did not display significant higher in vivo therapy efficacy than the free drug, the prodrug reduced the side toxicity. In addition, this heparin shell could potentially reduce the risk of severe hemorrhagic complication during systemic administration of antitumor drugs.

Another example is the macromolecular prodrug of heparin-dexamethasone conjugates.[64] Heparin was activated using adipic dihydrazide, and then conjugated with the poorly water-soluble dexamethasone via an acid-labile hydrazone linkage. The conjugates were amphiphilic and could form a prodrug micelle system. Combination of dexamethasone and doxorubicin is often used to improve multiple myeloma treatment. Therefore, doxorubicin was loaded in the hydrophobic cores the heparin-dexamethasone micelles. The dual-drug combinational delivery was characterized by acid-sensitive release profiles. With these micelles, drugs antitumor activity and intracellular accumulation in vitro were also improved.

\section{Heparin-based prodrug-type probes for tumor imaging}

Proteases play important roles in various biological and pathological processes. As a case in point, tumor-associated proteases (TAPs) are involved in cancer progression, including tumor angiogenesis, invasion and metastasis.[65, 66] TAPs have been widely explored for their application as diagnostic and prognostic biomarkers and drug targets as well. In vivo detection and imaging of a specific TAP offer a potential method for early diagnosis of cancer. Therefore, to develop a sensitive and specific in vivo probe is a pressing need. 
Legumain is an asparaginyl endopeptidase, overexpressed in many types of solid tumors. Its close association with tumorigenesis has been well documented,[67] with its promising clinical value as diagnostic biomarker and therapeutic target. We previously reported that legumain is also involved in the progression from inflammation to malignant colon tumors, and its expression was found dramatic up-regulation in the late stage of inflammation and early stage of tumor development.[23]

Given the important role of legumain in tumorigenesis, we developed a prodrug-type, legumain-activatable hybrid nanoprobe for tumor imaging, based on the fluorescence resonance energy transfer (FRET) effect (Fig. 15).[68] The hybrid nanoprobe system was composed of two parts as described below.

1) Fluorophore part: quantum dots (QDs) modified with low-molecular-weight heparin (LH), termed as QD-LH.

2) Fluorescence quencher part: QSY21 (quencher dye) modified with a specific peptide, termed as QSY21-PEP. The peptide contains two sequences: legumain-substrate peptide and low-molecularweight protamine (LP).

Via the strong charge interaction and affinity of LH and LP, these two parts can self-assemble together and form a hybrid nanoparticles, which is characterized by the surface modification of QSY21-PEP on the QD-LH. Due to the FRET effect, the nanoprobe is in an inactivated (or quenched) status. Once reaching the tumor, the overexpressed legumain would cleave the substrate peptide linker between QSY21 and LP, resulting the detachment of QSY21 from the system and consequently activating the fluorescence.

Of interest, the real-time activation process in a living cell was monitored using confocal microscopy. Fluorescence was initially activated on the membrane, and then the fluorescence intensity increased (Fig. 16). It should be noted that the formation of active legumain is a $\mathrm{pH}$-dependent process in the endosome/lysosome system, and the mature legumain then is transported onto the cell membrane and released into the extracellular space.

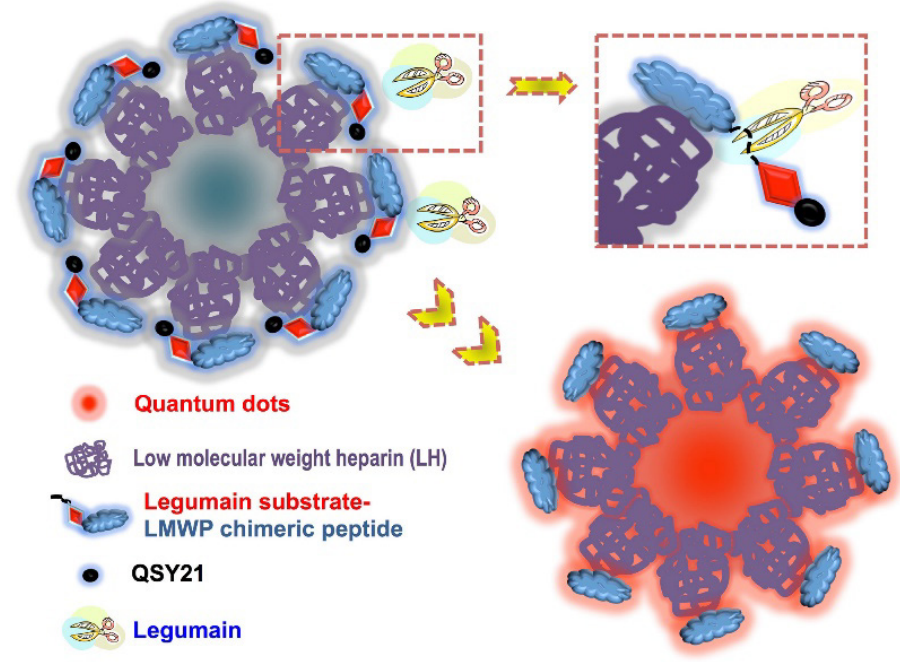

Fig. 15 The schematic illustration of the legumain-activatable nanoprobe for tumor imaging. Reproduced with permission from [68].

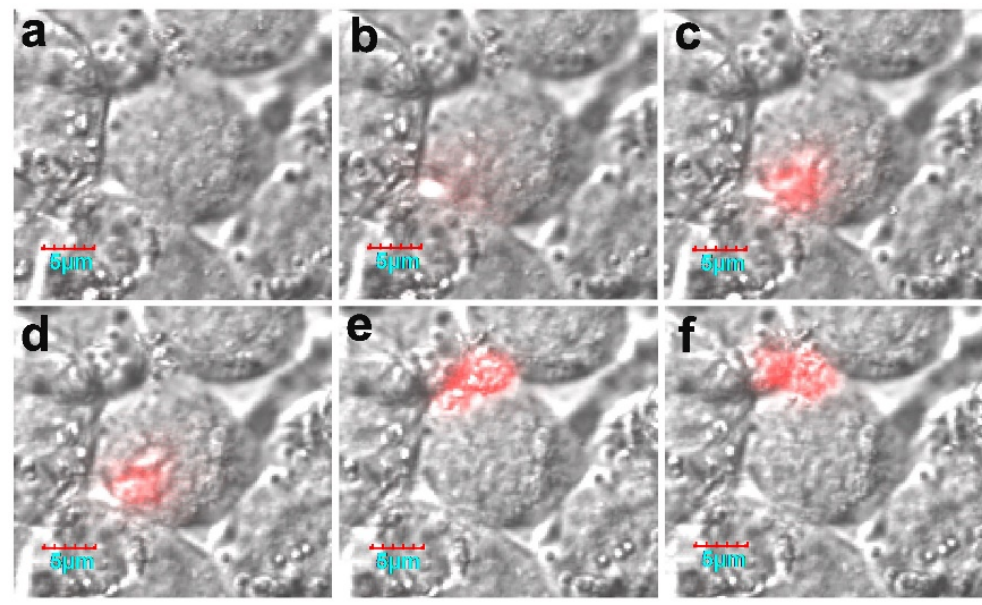

Fig. 16 The cellular activation process of the nanoprobe. Reproduced with permission from [68]. 
A great advantage of this hybrid system is no need of chemical conjugation of a fluorophore to a quencher. The use of the non-covalent linkage between LH/LP can ease the production. Of importance, heparin/protamine binding affinity is strong and no endogenous components can dissociate them, rendering their linkage very stable in vivo. Moreover, by simple replacement of the quencher part containing another protease substrate sequence, the nanoprobe can apply for detection of another TAP. It may develop into a potential platform technique.

With the similar design, we also developed a tumor-associated MMP targeted nanoprobe for tumor imaging. For this application, the MMP substrate peptide was used instead in the fluorescence quencher part. This system was evaluated in various subcutaneous fibrosarcoma, breast, and glioma tumor xenograft models that overexpress MMPs in tumor tissues, and it worked in all those tested models.[69] For instance, the fluorescence activation in tumor was fast (within $0.5 \mathrm{~h}$ ), and the signal in tumor was highest among other major tissues (Fig. 17), indicating the tumor-targeted imaging function. Similar results were found in MCF-7 breast tumor and U87 giloma models. Fig. 18 shows intense fluorescence was activated in HT1080 fibrosarcoma tissue slices. The nanoprobes distributed close to the tumor blood vessels (green), suggesting the possible mechanism of EPR effect. By contrast, very minor activation was observed in the SW620 colon tumor with low MMP-2 expression.

We found MMP-2 was highly expressed not only in the subcutaneous U87 tumor but also the orthotopic tumor. Actually, MMP-2 has been demonstrated its involvement in various progressions of glioma.[70] Yet the level in normal brain tissues was very low. Therefore, brain MMP-2 could be a potential target for brain tumor detection and imaging. Due to the formidable blood-brain barrier (BBB), brain delivery of a nanoprobe is challenging. To address this issue, a brain-targeted sequence (HAIYPRH, termed T7) was introduced to the activatable MMP substrate peptide-LMWP (PLGVRGGVSRRRRRRGGRRRR, termed ALMWP). A multifunctional hybrid peptide (T7-(QSY21)KGG-ALMWP) was synthesized, and the quencher QSY21 was conjugated to the $\varepsilon$-amine of lysine. $\mathrm{T} 7$ is a targeting ligand to transferrin receptors that are overexpressed in the BBB, and importantly, its binding site on transferrin receptors is distinct from the endogenous transferrin binding site.[71] It has been used in various nanoparticulate systems for brain tumor delivery.[72-74] Consistent with those previous reports, the T7-(QSY21)-ALMWP/LH-QD system was found to be able to penetrate the BBB and accumulate inside the glioma (Fig. 19A). The fluorescence intensity gradually increased in $10 \mathrm{~h}$, and a declination was found at $22 \mathrm{~h}$ (Fig. 19B).
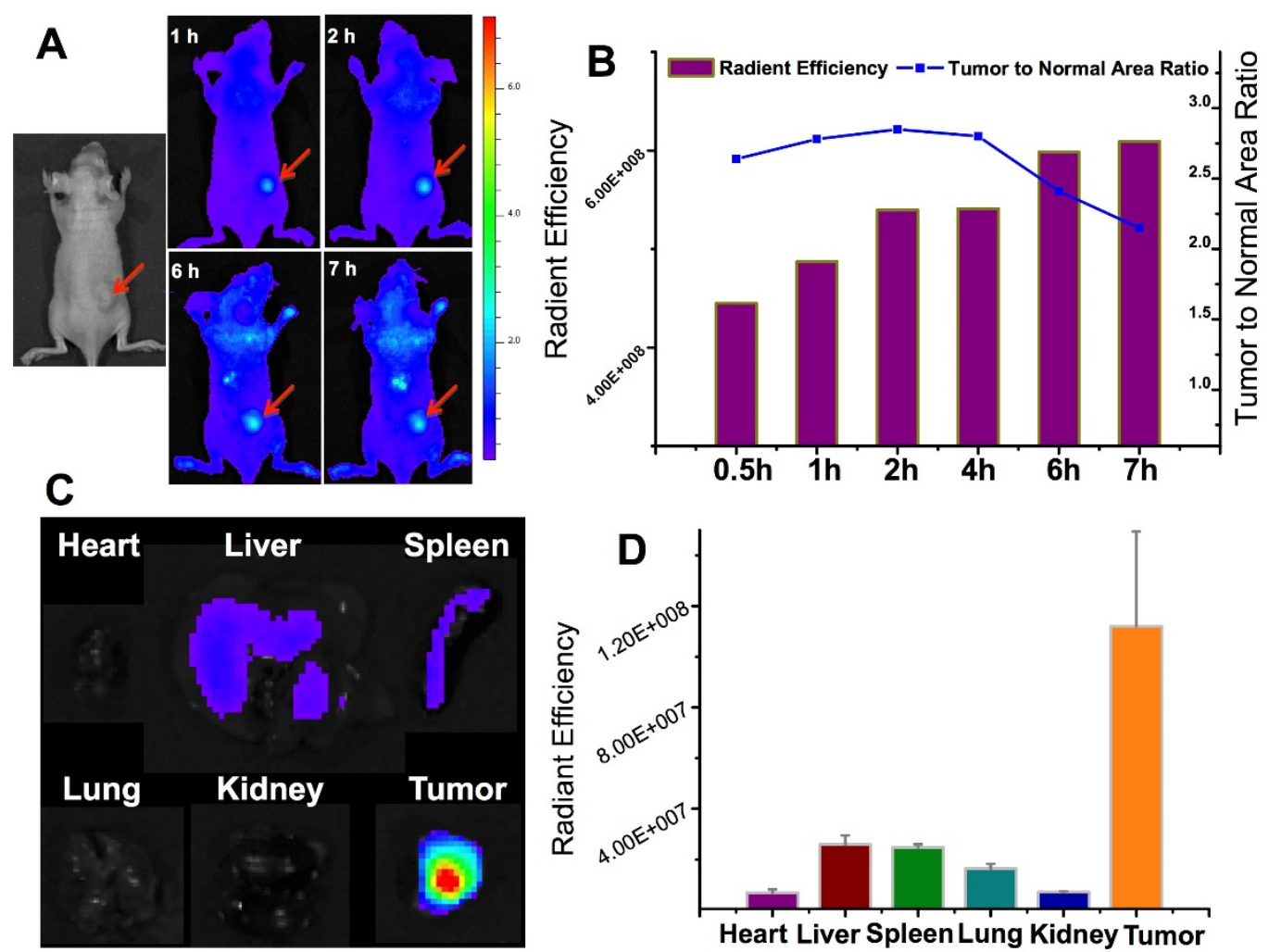

Fig. 17 The in vivo imaging of the MMP-activatable nanoprobe in the subcutaneous HT1080 tumor model. Reproduced with permission from [69]. 

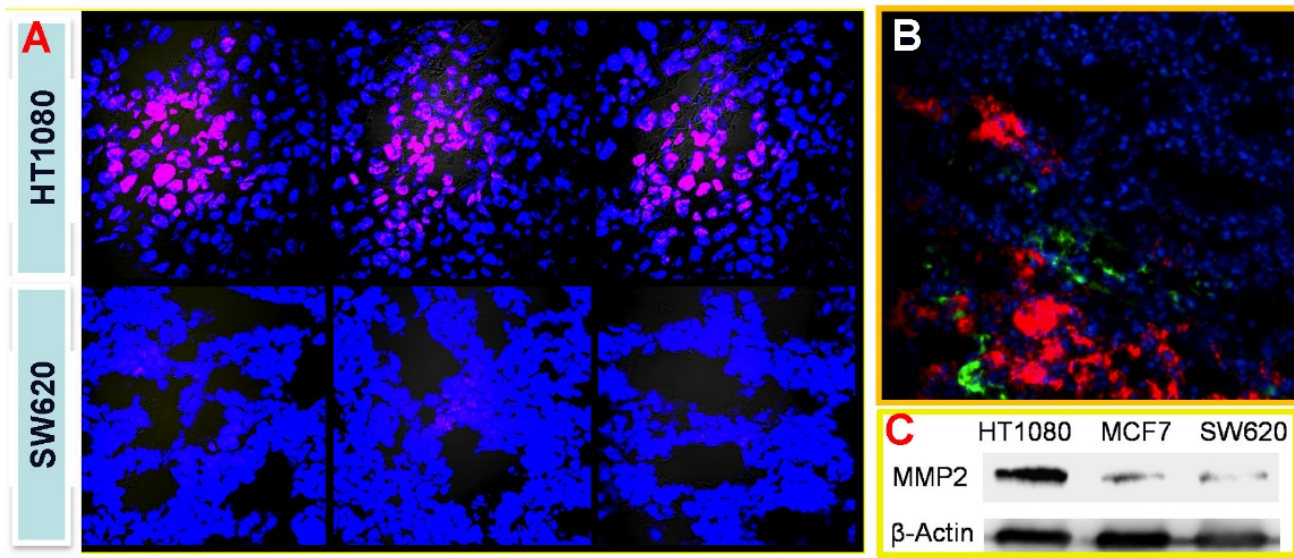

Fig. 18 (A) Tumor-associated activation of the nanoprobe. (B) Blood vessel staining (green) and the activated nanoprobe (red) in HT1080 tumor. (C) Western blotting analysis of MMP-2 in HT1080, MCF-7, and SW620 tumor. Reproduced with permission from [69].

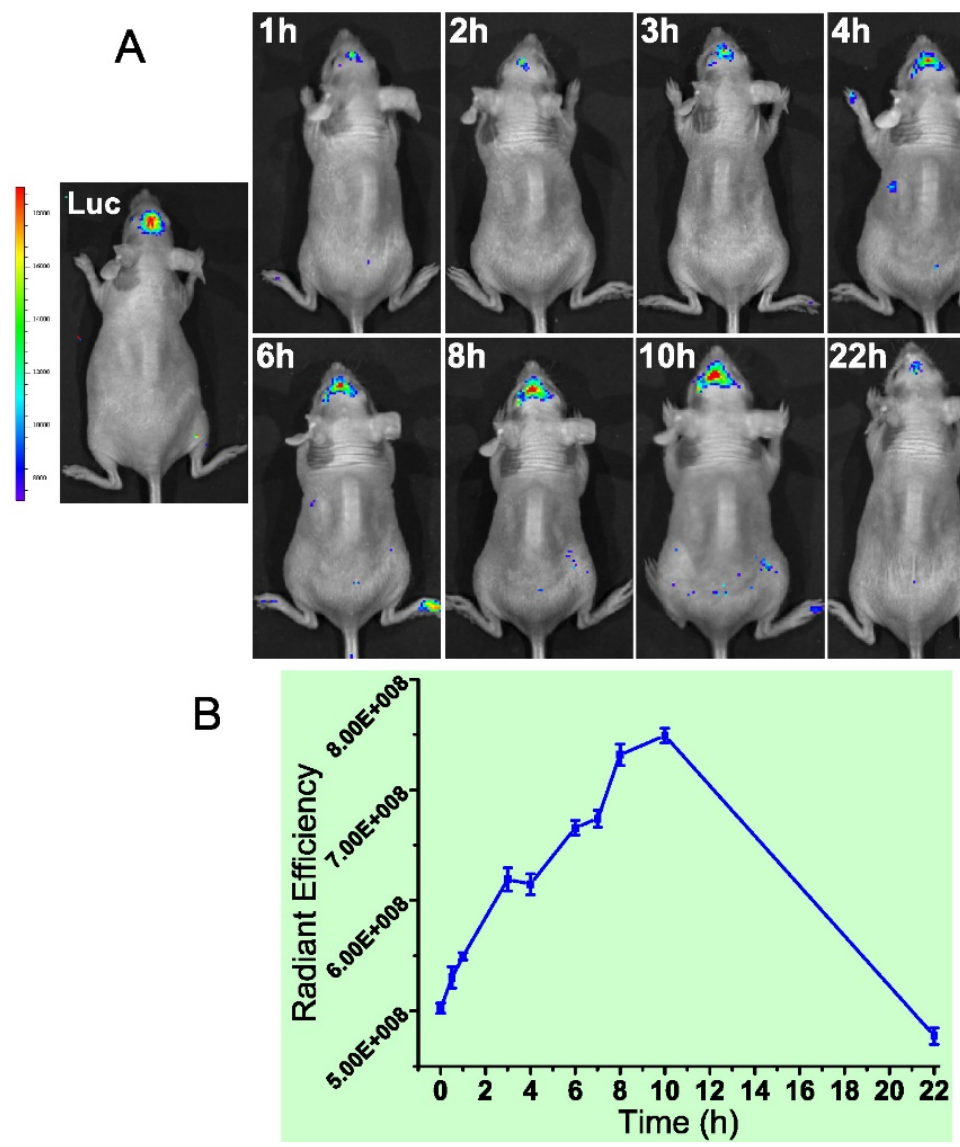

Fig. 19 (A) The orthotopic glioma imaging with the T7-functionalized nanoprobe at various time points. (B) The radiant efficiency (fluorescence intensity). Reproduced with permission from [69].

\section{Heparin derivative-based nano drug delivery systems for cancer therapy}

It has been reported that low-molecular-weight heparin derivatives possess high anti-angiogenic but low anti-coagulant effects.[75] Byun et al developed a low-molecular-weight heparin-taurocholate conjugate (LHT7), which showed a significant anti-angiogenic activity with negligible anticoagulant activity due to its lower binding affinity to antithrombin III but higher binding affinity to multiple angiogenic growth factors than LMWH does.[76] A liposomal co-delivery system of LHT7 and suberanilohydroxamic acid (SAHA), a histone deacetylase inhibitor, named LHT7/SAHA nanolipoplex, was prepared by encapsulating slightly soluble SAHA inside cationic nanolipoplex, followed by coating of negative charge LHT7 onto the cationic surfaces by charge-charge interaction.[77] LHT7/SAHA nanolipoplexes showed for enhanced tumor vasculature targeting and 
improved therapeutic efficacy in the SCC7-bearing mice model with obviously decreased tumor weight and volume (Fig. 20). Furthermore, this group prepared the reduced graphene oxide (rGO) nanosheets coated with LHT7 as a tumor-targeting nanodelivery system for anticancer therapy that could delivery chemotherapy drugs (e.g., DOX).[78] LHT-rGO showed a higher Dox loading capacity compared with uncoated rGO. The in vivo tumor tissue distribution and retention and antitumor efficacy of LHT-rGO/Dox was evaluated in KB tumor bearing mice. The drug accumulation was greater in LHT-rGO/Dox-treated group than in rGO/Dox-treated group. At $24 \mathrm{~h}$ post dose, the tumor accumulation of LHT-rGO/Dox was 7-fold higher than that of rGO/Dox. The tumor volumes and tumor weights showed that the enhanced therapy effect of LHT-rGO/Dox over the other groups was observed with the reduced tumor weight rate of $92.5 \%$. The in vivo anti-tumor effect might also be partially explained by the anti-angiogenic and anticancer effect of LHT7 by binding with angiogenic growth factors which are over-expressed in tumor micro-environment, e.g., vascular endothelial growth factor, basic fibroblast growth factor, and platelet-derived endothelial cell growth factor.
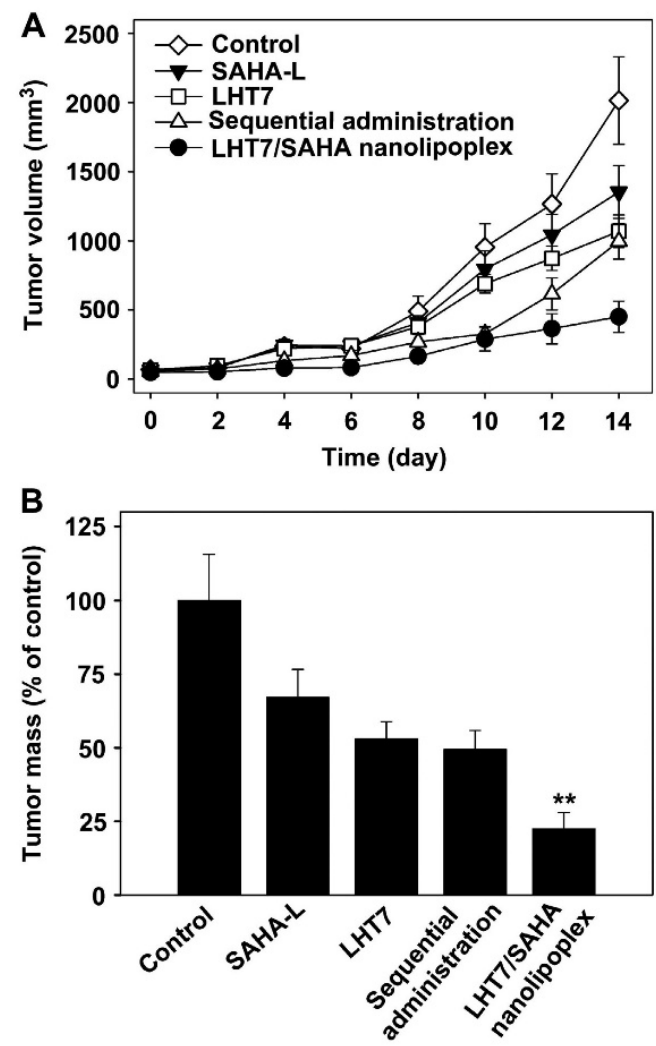

Fig. 20 (A) The tumor volume of PBS, SAHA-L, LHT7, the sequential co-administration of SAHA-L and LHT7, or LHT7/SAHA nanolipoplex groups treated. (B) Comparative tumor mass after 14 days. Reproduced with permission from [72]

\section{Concluding remarks}

There are a large variety of protein drugs that lack organ-specific action, thereby causing side effects. Prodrug-type delivery systems provide a promising solution to achieve targeted delivery of protein drugs. CPP-mediated macromolecular delivery still remains formidable challenges for in vivo application due to its non-specific organ distribution and penetration. On the other hand, CPP has been a well-established, potent tool for enhanced macromolecular delivery. In order to make good use of the potent penetration ability, while curbing its nonselectivity, it should manage a balancing act between the two sides of the dilemma. A series of investigations have delved deeply into this issue in our groups by employing the heparin-regulated strategy. Heparin-regulated prodrug delivery mechanisms include non-covalent linkage by heparin-CPP electrostatic interaction, reversible inhibition to the cell-penetration ability by heparin, and protamine-triggered drug release. The regulation is simple and highly controllable. Compared to the microenvironment-responsive mechanism, the heparin-regulated strategy is direct and instantly responsive to the given dose of protamine, yet independent to real-time pathological conditions. Therefore, this method offers a potential platform for targeted delivery of various drugs.

It also should be pointed out that heparin, widely used as the regulation material in "ATTEMPTS" for anticancer therapy, has high anti-coagulant activity, which might set limits to its clinical application. Overdose of heparin often causes unexpected adverse effects such as bleeding and heparin-induced thrombocytopenia. Once heparin is free from the ATTEMPTS, there could be side effects. To eliminate such a potential risk, the timing for giving the trigger agent protamine is important. It is ideal that protamine should be administered when drug concentration peaks at tumor, while that in circulation drops to an insignificant level. Therefore, the determination of trigger timing could be a crucial issue that influences the clinical translation of this system. Better understanding of the pharmacokinetics profiles of the system will be helpful to predict the trigger timing. In addition, we are optimistic that the advance of real-time in vivo imaging techniques will also provide a use tool for timely dosing.

\section{Acknowledgements}

We thank the support from National Basic Research Program of China (973 Program 2014CB931900, 2013CB93250) and NSFC, China 
(81373357,

81361140344

81422048

81402883 81673382).

\section{Competing Interests}

The authors have declared that no competing interest exists.

\section{References}

1. Lee YC, Lee RT. Carbohydrate-Protein Interactions - Basis of Glycobiology. Accounts of Chemical Research. 1995; 28: 321-7.

2. Powell AK, Yates EA, Fernig DG, Turnbull JE. Interactions of heparin/heparan sulfate with proteins: appraisal of structural factors and experimental approaches. Glycobiology. 2004; 14: 17R-30R.

3. Capila I, Linhardt RJ. Heparin-protein interactions. Angew Chem Int Ed Engl. 2002; 41: 391-412.

4. George CM. Thromboembolic Disease. In: Helms RA, Quan DJ, editors. Textbook of therapeutics: drug and disease management. 8th ed. Philadelphia: Lippincott Williams \& Wilkins; 2006: 634.

5. Mecca T, Consoli GM, Geraci C, La Spina R, Cunsolo F. Polycationic calix[8]arenes able to recognize and neutralize heparin. Org Biomol Chem. 2006; 4: 3763-8.

6. Sapra P, Stein R, Pickett J, Qu Z, Govindan SV, Cardillo TM, et al. Anti-CD74 antibody-doxorubicin conjugate, IMMU-110, in a human multiple myeloma xenograft and in monkeys. Clin Cancer Res. 2005; 11: 5257-64.

7. Guillemard V, Saragovi HU. Taxane-antibody conjugates afford potent cytotoxicity, enhanced solubility, and tumor target selectivity. Cancer Res. 2001; 61: 694-9.

8. Kreitman RJ. Immunotoxins for targeted cancer therapy. AAPS J. 2006; 8: E532-51.

9. Walker I, Irwin WJ, Akhtar S. Improved cellular delivery of antisense oligonucleotides using transferrin receptor antibody-oligonucleotide conjugates. Pharm Res. 1995; 12: 1548-53.

10. Khalil IA, Kogure K, Akita H, Harashima H. Uptake pathways and subsequent intracellular trafficking in nonviral gene delivery. Pharmacol Rev. 2006; 58: $32-45$

11. Rautio J, Kumpulainen H, Heimbach T, Oliyai R, Oh D, Jarvinen T, et al. Prodrugs: design and clinical applications. Nat Rev Drug Discov. 2008; 7: 255-70.

12. Byun Y, Yang VC. Delivery system for targeted thrombolysis without the risk of hemorrhage. ASAIO J. 1998; 44: M638-41.

13. Song $\mathrm{H}$, Liang JF, Yang VC. A prodrug approach for delivery of t-PA: construction of the cationic t-PA prodrug by a recombinant method and preliminary in vitro evaluation of the construct. ASAIO J. 2000; 46: 663-8.

14. Liang JF, Li YT, Song H, Park YJ, Naik SS, Yang VC. ATTEMPTS: a heparin/protamine-based delivery system for enzyme drugs. J Control Release. 2002; 78: 67-79.

15. Liang JF, Park YJ, Song H, Li YT, Yang VC. ATTEMPTS: a heparin/protamine-based prodrug approach for delivery of thrombolytic drugs. J Control Release. 2001; 72: 145-56.

16. Park YJ, Liang JF, Song H, Li YT, Naik S, Yang VC. ATTEMPTS: a heparin/protamine-based triggered release system for the delivery of enzyme drugs without associated side-effects. Adv Drug Deliv Rev. 2003; 55: 251-65.

17. Liang JF, Li YT, Connell ME, Yang VC. Synthesis and characterization of positively charged tPA as a prodrug using heparin/protamine-based drug delivery system. AAPS PharmSci. 2000; 2: E7.

18. Yang VC, Naik SS, Song H, Dombkowski AA, Crippen G, Liang JF. Construction and characterization of a t-PA mutant for use in ATTEMPTS: a drug delivery system for achieving targeted thrombolysis. J Control Release. 2005; 110: 164-76.

19. Liu X, Xiang J, Zhu D, Jiang L, Zhou Z, Tang J, et al. Fusogenic Reactive Oxygen Species Triggered Charge-Reversal Vector for Effective Gene Delivery. Adv Mater. 2016; 28: 1743-52.

20. Qiu N, Liu X, Zhong Y, Zhou Z, Piao Y, Miao L, et al. Esterase-Activated Charge-Reversal Polymer for Fibroblast-Exempt Cancer Gene Therapy. Adv Mater. 2016; 28: 10613-22.

21. Chen $Y$, Zhang $M$, Jin $H$, Tang $Y$, Wang $H, X u Q$, et al. Intein-mediated site-specific synthesis of tumor-targeting protein delivery system: Turning PEG dilemma into prodrug-like feature. Biomaterials. 2017; 116: 57-68.

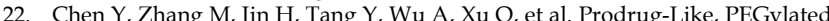
Protein Toxin Trichosanthin for Reversal of Chemoresistance. Molecular Pharmaceutics. 2017

23. Jiang $\mathrm{Y}$, Lu J, Wang $\mathrm{Y}$, Zeng $\mathrm{F}$, Wang $\mathrm{H}$, Peng $\mathrm{H}$, et al. Molecular-dynamics-simulation-driven design of a protease-responsive probe for in-vivo tumor imaging. Adv Mater. 2014; 26: 8174-8.

24. Naik SS, Liang JF, Park YJ, Lee WK, Yang VC. Application of "ATTEMPTS" for drug delivery. J Control Release. 2005; 101: 35-45.

25. Absar S, Choi S, Yang VC, Kwon YM. Heparin-triggered release of camouflaged tissue plasminogen activator for targeted thrombolysis. J Control Release. 2012; 157: 46-54.
26. Huang $Y$, Jiang $Y$, Wang $H$, Wang J, Shin MC, Byun $Y$, et al Curb challenges of the "Trojan Horse" approach: Smart strategies in achieving effective yet safe cell-penetrating peptide-based drug delivery. Adv Drug Deliv Rev. 2013; 65: 1299-315.

27. Koren E, Torchilin VP. Cell-penetrating peptides: breaking through to the other side. Trends Mol Med. 2012; 18: 385-93.

28. Lin $\mathrm{T}$, Zhao $\mathrm{P}$, Jiang $\mathrm{Y}$, Tang $\mathrm{Y}$, Jin $\mathrm{H}$, Pan $\mathrm{Z}$, et al Blood-Brain-Barrier-Penetrating Albumin Nanoparticles for Biomimetic Drug Delivery via Albumin-Binding Protein Pathways for Antiglioma Therapy. ACS Nano. 2016; 10: 9999-10012.

29. Lin T, Liu E, He H, Shin MC, Moon C, Yang VC, et al. Nose-to-brain delivery of macromolecules mediated by cell-penetrating peptides. Acta Pharm Sin B. 2016; 6: 352-8

30. Yang YX, Jiang YF, Wang Z, Liu JH, Yan L, Ye JX, et al. Skin-permeable quaternary nanoparticles with layer-by-layer structure enabling improved gene delivery. J Mater Chem. 2012; 22: 10029-34.

31. Huang Y, Park YS, Moon C, David AE, Chung HS, Yang VC. Synthetic skin-permeable proteins enabling needleless immunization. Angew Chem Int Ed Engl. 2010; 49: 2724-7.

32. Gao W, Xiang B, Meng TT, Liu F, Qi XR. Chemotherapeutic drug delivery to cancer cells using a combination of folate targeting and tumor microenvironment-sensitive polypeptides. Biomaterials. 2013; 34: 4137-49.

33. Console S, Marty C, Garcia-Echeverria C, Schwendener R, Ballmer-Hofer K. Antennapedia and HIV transactivator of transcription (TAT) "protein transduction domains" promote endocytosis of high molecular weight cargo upon binding to cell surface glycosaminoglycans. J Biol Chem. 2003; 278: 35109-14.

34. Heitz F, Morris MC, Divita G. Twenty years of cell-penetrating peptides: from molecular mechanisms to therapeutics. Br J Pharmacol. 2009; 157: 195-206.

35. Mann DA, Frankel AD. Endocytosis and targeting of exogenous HIV-1 Tat protein. EMBO J. 1991; 10: 1733-9.

36. Ziegler A, Seelig J. Interaction of the protein transduction domain of HIV-1 TAT with heparan sulfate: binding mechanism and thermodynamic parameters. Biophys J. 2004; 86: 254-63.

37. Tyagi M, Rusnati M, Presta M, Giacca M. Internalization of HIV-1 tat requires cell surface heparan sulfate proteoglycans. J Biol Chem. 2001; 276: 3254-61.

38. Hakansson S, Jacobs A, Caffrey M. Heparin binding by the HIV-1 tat protein transduction domain. Protein Sci. 2001; 10: 2138-9.

39. Hakansson S, Caffrey M. Structural and dynamic properties of the HIV-1 tat transduction domain in the free and heparin-bound states. Biochemistry. 2003; 42: 8999-9006

40. Ziegler A. Thermodynamic studies and binding mechanisms of cell-penetrating peptides with lipids and glycosaminoglycans. Adv Drug Deliv Rev. 2008; 60: 580-97.

41. Sawant RR, Torchilin VP. Enhanced cytotoxicity of TATp-bearing paclitaxel-loaded micelles in vitro and in vivo. Int J Pharm. 2009; 374: 114-8

42. Chang LC, Lee HF, Yang Z, Yang VC. Low molecular weight protamine (LMWP) as nontoxic heparin/low molecular weight heparin antidote (I): preparation and characterization. AAPS PharmSci. 2001; 3: E17.

43. He H, David AE, Zhang J, Park YS, Wang J, Huang Y, et al. Low molecular weight protamine/insulin formulation with potential to attenuate protamine-masqueraded insulin allergy. Macromol Res. 2011; 19: 1224-6.

44. Park YS, Huang Y, Park YJ, David AE, White L, He H, et al. Specific down regulation of 3T3-L1 adipocyte differentiation by cell-permeable antisense HIF1alpha-oligonucleotide. J Control Release. 2010.

45. Park YS, David AE, Huang Y, Park JB, He H, Byun Y, et al. In vivo delivery of cell-permeable antisense hypoxia-inducible factor 1alpha oligonucleotide to adipose tissue reduces adiposity in obese mice. J Control Release. 2012; 161: $1-9$.

46. Wang $\mathrm{H}$, Zhao $\mathrm{Y}$, Wang $\mathrm{H}$, Gong J, He $\mathrm{H}$, Shin $\mathrm{MC}$, et al. Low-molecular-weight protamine-modified PLGA nanoparticles for overcoming drug-resistant breast cancer. J Control Release. 2014; 192C: 47-56.

47. Lee LM, Chang LC, Wrobleski S, Wakefield TW, Yang VC. Low molecular weight protamine as nontoxic heparin/low molecular weight heparin antidote (III): preliminary in vivo evaluation of efficacy and toxicity using a canine model. AAPS PharmSci. 2001; 3: E19.

48. Chang LC, Liang JF, Lee HF, Lee LM, Yang VC. Low molecular weight protamine (LMWP) as nontoxic heparin/low molecular weight heparin antidote (II): in vitro evaluation of efficacy and toxicity. AAPS PharmSci. 2001; 3: E18.

49. Shin MC, Zhang J, David AE, Trommer WE, Kwon YM, Min KA, et al. Chemically and biologically synthesized CPP-modified gelonin for enhanced anti-tumor activity. J Control Release. 2013; 172: 169-78

50. Shin MC, Zhang J, Min KA, Lee K, Moon C, Balthasar JP, et al. Combination of antibody targeting and PTD-mediated intracellular toxin delivery for colorectal cancer therapy. J Control Release. 2014; 194: 197-210.

51. Shin MC, Zhao J, Zhang J, Huang Y, He H, Wang M, et al. Recombinant TAT-gelonin fusion toxin: synthesis and characterization of heparin/protamine-regulated cell transduction. J Biomed Mater Res A. 2015; 103: 409-19.

52. Tiernan JP, Perry SL, Verghese ET, West NP, Yeluri S, Jayne DG, et al. Carcinoembryonic antigen is the preferred biomarker for in vivo colorectal cancer targeting. Br J Cancer. 2013; 108: 662-7. 
53. Tiernan JP, Ingram N, Marston G, Perry SL, Rushworth JV, Coletta PL, et al. CEA-targeted nanoparticles allow specific in vivo fluorescent imaging of colorectal cancer models. Nanomedicine (Lond). 2015; 10: 1223-31.

54. Urva SR, Balthasar JP. Target mediated disposition of T84.66, a monoclonal anti-CEA antibody: application in the detection of colorectal cancer xenografts. MAbs. 2010; 2: 67-72.

55. Shin MC, Zhang J, Min KA, He H, David AE, Huang Y, et al. PTD-Modified ATTEMPTS for Enhanced Toxin-based Cancer Therapy: An In Vivo Proof-of-Concept Study. Pharm Res. 2015; 32: 2690-703.

56. Avramis VI, Panosyan EH. Pharmacokinetic/pharmacodynamic relationships of asparaginase formulations: the past, the present and recommendations for the future. Clin Pharmacokinet. 2005; 44: 367-93.

57. Kwon YM, Li YT, Liang JF, Park YJ, Chang LC, Yang VC. PTD-modified ATTEMPTS system for enhanced asparaginase therapy: a proof-of-concept investigation. J Control Release. 2008; 130: 252-8.

58. Li YT, Kwon YM, Spangrude GJ, Liang JF, Chung HS, Park YJ, et al. Preliminary in vivo evaluation of the protein transduction domain-modified ATTEMPTS approach in enhancing asparaginase therapy. J Biomed Mater Res A. 2009; 91: 209-20.

59. Fang J, Nakamura H, Maeda H. The EPR effect: Unique features of tumor blood vessels for drug delivery, factors involved, and limitations and augmentation of the effect. Adv Drug Deliv Rev. 2011; 63: 136-51.

60. Li C, Wallace S. Polymer-drug conjugates: recent development in clinical oncology. Adv Drug Deliv Rev. 2008; 60: 886-98.

61. Moon C, Kwon YM, Lee WK, Park YJ, Chang LC, Yang VC. A novel polyrotaxane-based intracellular delivery system for camptothecin: in vitro feasibility evaluation. J Biomed Mater Res A. 2008; 84: 238-46.

62. Moon C, Kwon YM, Lee WK, Park YJ, Yang VC. In vitro assessment of a novel polyrotaxane-based drug delivery system integrated with a cell-penetrating peptide. J Control Release. 2007; 124: 43-50.

63. Wang Y, Xin D, Liu K, Zhu M, Xiang J. Heparin-paclitaxel conjugates as drug delivery system: synthesis, self-assembly property, drug release, and antitumor activity. Bioconjug Chem. 2009; 20: 2214-21.

64. Li NN, Lin J, Gao D, Zhang LM. A macromolecular prodrug strategy for combinatorial drug delivery. J Colloid Interface Sci. 2014; 417: 301-9.

65. Lopez-Otin C, Bond JS. Proteases: multifunctional enzymes in life and disease. J Biol Chem. 2008; 283: 30433-7.

66. Mason SD, Joyce JA. Proteolytic networks in cancer. Trends Cell Biol. 2011; 21: 228-37.

67. Liu C, Sun C, Huang H, Janda K, Edgington T. Overexpression of legumain in tumors is significant for invasion/metastasis and a candidate enzymatic target for prodrug therapy. Cancer Res. 2003; 63: 2957-64.

68. Wang Y, Jiang Y, Zhang M, Tan J, Liang J, Wang H, et al. Protease-Activatable Hybrid Nanoprobe for Tumor Imaging. Adv Funct Mater. 2014; 24: 5443-53.

69. Wang $\mathrm{Y}$, Lin $\mathrm{T}$, Zhang $\mathrm{W}$, Jiang $\mathrm{Y}$, Jin $\mathrm{H}, \mathrm{He} \mathrm{H}$, et al. A Prodrug-type, MMP-2-targeting Nanoprobe for Tumor Detection and Imaging. Theranostics. 2015; 5: 787-95.

70. Badiga AV, Chetty C, Kesanakurti D, Are D, Gujrati M, Klopfenstein JD, et al. MMP-2 siRNA inhibits radiation-enhanced invasiveness in glioma cells. PLoS One. 2011; 6: e20614.

71. Lee JH, Engler JA, Collawn JF, Moore BA. Receptor mediated uptake of peptides that bind the human transferrin receptor. Eur J Biochem. 2001; 268: 2004-12.

72. Cui YN, Zhang M, Zeng F, Jin HY, Xu Q, Huang YZ. Dual-targeting magnetic PLGA nanoparticles for codelivery of paclitaxel and curcumin for brain tumor therapy. ACS Appl Mater Interfaces. 2016; 8: 32159-69.

73. Zong T, Mei L, Gao H, Cai W, Zhu P, Shi K, et al. Synergistic dual-ligand doxorubicin liposomes improve targeting and therapeutic efficacy of brain glioma in animals. Mol Pharm. 2014; 11: 2346-57.

74. Gao H. Progress and perspectives on targeting nanoparticles for brain drug delivery. Acta Pharm Sin B. 2016; 6: 268-86.

75. Park J, Byun Y. Recent advances in anticoagulant drug delivery. Expert Opin Drug Deliv. 2016; 13: 421-34.

76. Chung SW, Bae SM, Lee M, Al-Hilal TA, Lee CK, Kim JK, et al. LHT7, a chemically modified heparin, inhibits multiple stages of angiogenesis by blocking VEGF, FGF2 and PDGF-B signaling pathways. Biomaterials. 2015; 37: 271-8.

77. Kim JY, Shim G, Choi HW, Park J, Chung SW, Kim S, et al. Tumor vasculature targeting following co-delivery of heparin-taurocholate conjugate and suberoylanilide hydroxamic acid using cationic nanolipoplex. Biomaterials. 2012; 33: 4424-30.

78. Shim G, Kim JY, Han J, Chung SW, Lee S, Byun Y, Oh YK. Reduced graphene oxide nanosheets coated with an anti-angiogenic anticancer low-molecular-weight heparin derivative for delivery of anticancer drugs. J Control Release. 2014; 189: 80-89. 